\title{
Springs, Streams, and Gas Vent on and Near Mount Adams Volcano, Washington
}

By Manuel Nathenson and Robert H. Mariner

Scientific Investigations Report 2013-5097 


\title{
U.S. Department of the Interior SALLY JEWELL, Secretary
}

\section{U.S. Geological Survey \\ Suzette M. Kimball, Acting Director}

\author{
U.S. Geological Survey, Reston, Virginia: 2013
}

For product and ordering information: World Wide Web: http://www.usgs.gov/pubprod Telephone: 1-888-ASK-USGS

For more information on the USGS - the Federal source for science about the Earth, its natural and living resources, natural hazards, and the environment:

World Wide Web: http://www.usgs.gov

Telephone: 1-888-ASK-USGS

Suggested citation:

Nathenson, Manuel, and Mariner, R.H., 2013, Springs, streams, and gas vent on and near Mount Adams volcano, Washington: U.S. Geological Survey Scientific Investigations Report 2013-5097, 19 p., [Available on the Web at http://pubs.usgs.gov/sir/2013/5097/.]

Any use of trade, product, or firm names is for descriptive purposes only and does not imply endorsement by the U.S. Government.

Although this information product, for the most part, is in the public domain, it also may contain copyrighted materials as noted in the text. Permission to reproduce copyrighted items must be secured from the copyright owner. 


\section{Contents}

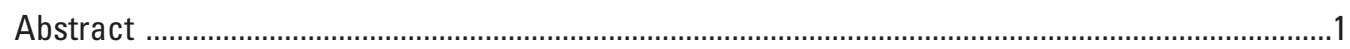

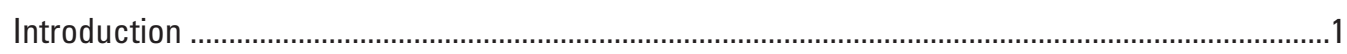

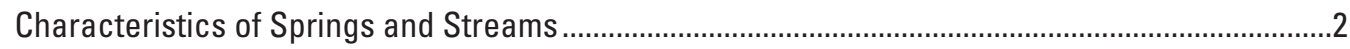

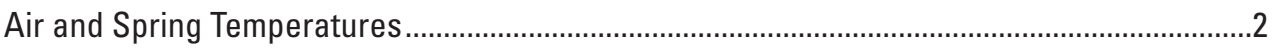

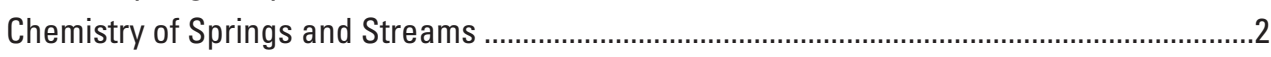

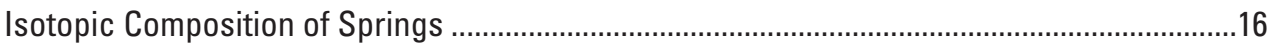

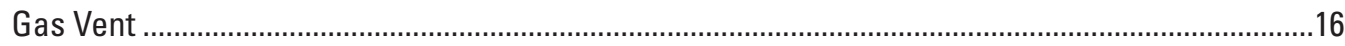

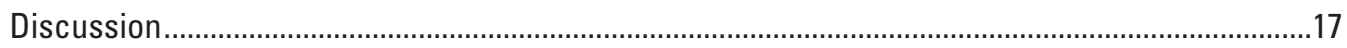

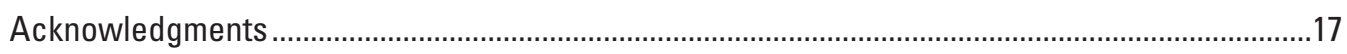

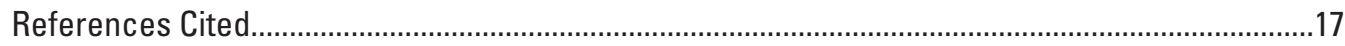

\section{Figures}

1. Map of Mount Adams, Washington, showing locations of sites visited in this study and those

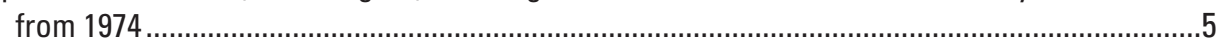

2. Map of weather stations in the vicinity of Mount Adams with 30-year records for calculation of normal air temperatures ..........................................................................................

3. Graph of temperature versus elevation for air temperatures from weather stations shown in figure 2 and for temperatures of springs from sites in figure 1 ............................................

4. Graph of sodium versus bicarbonate concentrations for springs and streams in the Mount

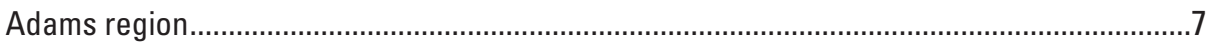

5. Graph of silica concentration versus specific conductance for springs and streams in the Mount Adams region....................................................................................................

6. Graph of calcium versus sulfate concentrations for springs and streams in the Mount

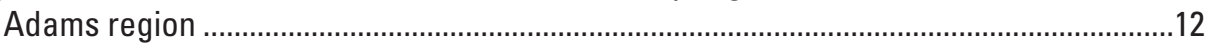

7. Photo of Cascade Creek at the location of sampling, showing the reddish-brown color of

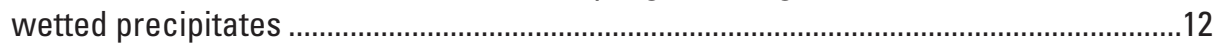

8. Graph of deuterium/hydrogen deviation $(\delta D)$ versus oxygen-18/oxygen-16 deviation $\left(\delta^{18} 0\right)$ for water samples from table 2...............................................................................13

9. Graph of oxygen-18/oxygen-16 ratio deviation versus elevation for samples from table 2...13

10. Triangular diagram showing relative proportions of nitrogen, argon, and helium for gas data

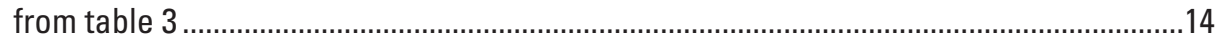

11. Triangular diagram showing relative proportions of methane, argon, and helium for gas data

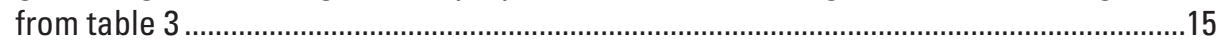

\section{Tables}

1. Physical measurements and sampling information on springs and streams on and near Mount Adams. ............................................................................................................

2. Chemical and isotopic data for water samples from springs and streams on and near Mount

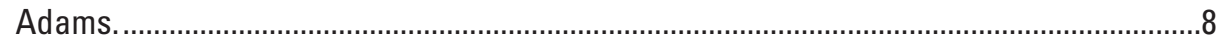

3. Chemical analysis of gas sample from the vent on Mount Adams, along with data for selected samples from Cascade volcanoes. 
This page left intentionally blank. 


\title{
Springs, Streams, and Gas Vent on and Near Mount Adams Volcano, Washington
}

\author{
By Manuel Nathenson and Robert H. Mariner
}

\section{Abstract}

Springs and some streams on Mount Adams volcano have been sampled for chemistry and light stable isotopes of water. Spring temperatures are generally cooler than air temperatures from weather stations at the same elevation. Spring chemistry generally reflects weathering of volcanic rock from dissolved carbon dioxide. Water in some springs and streams has either dissolved hydrothermal minerals or has reacted with them to add sulfate to the water. Some samples appear to have obtained their sulfate from dissolution of gypsum while some probably involve reaction with sulfide minerals such as pyrite. Light stable isotope data for water from springs follow a local meteoric water line, and the variation of isotopes with elevation indicate that some springs have very local recharge and others have water from elevations a few hundred meters higher. No evidence was found for thermal or slightly thermal springs on Mount Adams. A sample from a seeping gas vent on Mount Adams was at ambient temperature, but the gas is similar to that found on other Cascade volcanoes. Helium isotopes are 4.4 times the value in air, indicating that there is a significant component of mantle helium. The lack of fumaroles on Mount Adams and the ambient temperature of the gas indicates that the gas is from a hydrothermal system that is no longer active.

\section{Introduction}

Mount Adams stratovolcano in southwestern Washington covers an area of approximately $600 \mathrm{~km}^{2}$ and reaches an elevation of 3,742 $\mathrm{m}$ (Hildreth and Fierstein, 1995). An additional area of $650 \mathrm{~km}^{2}$ is covered by the surrounding Mount Adams volcanic field. Mount Adams lavas are predominantly andesitic with minor amounts of basalt and dacite, whereas the surrounding volcanic field is largely basaltic (Hildreth and Lanphere, 1994). Eruptions in the volcanic field started at about $950 \mathrm{ka}$, and construction of the Mount Adams edifice started at about $520 \mathrm{ka}$. The volume rate of eruption has varied episodically, with the most recent period of high production having built the current central edifice during the period 35 to $10 \mathrm{ka}$. Eruptions have continued into the Holocene, but the rate of eruption has not been large (Hildreth and Fierstein, 1997). The most recent eruptions of lava appear to be older than the 3,500-yr-BP Ye ash layer from Mount St. Helens, but some locally derived ash layers are younger than 2,500 yr BP but older than 1482 C.E., indicating that there have been more recent eruptions (Hildreth and Fierstein, 1995).

Stratovolcanoes with dominantly andesite lava products usually do not develop large upper crustal magma chambers that provide the heat source needed to sustain hydrothermal systems. However, it is estimated that an area of about $4 \mathrm{~km}^{2}$ on the summit cone of Mount Adams has undergone hydrothermal alteration, with an area of $0.55 \mathrm{~km}^{2}$ having undergone moderate to severe acid-sulfate alteration (Hildreth and Fierstein, 1995; Vallance, 1999). Data from sulfur prospecting suggests that there is an additional $0.54 \mathrm{~km}^{2}$ of extensively altered rock in an area covered by ice (Vallance, 1999). Hydrothermal alteration is exposed in cliffs and cleavers (a thin ridge of rock formed by the flow of two glaciers) extending 500-1,000 $\mathrm{m}$ below the summit, indicating that a volume of $0.9-3.3 \mathrm{~km}^{3}$ is altered (Vallance, 1999). Helicopter surveys for resistivity show that the summit area is underlain by low resistivity to an assumed penetration depth of $300 \mathrm{~m}$, and magnetic mapping indicates that nonmagnetic material from hydrothermal alteration extends to depths of 800-1,000 m (Finn and others, 2007), similar to the estimate based on exposures in cliffs and cleavers. The volume of low-resistivity and nonmagnetic regions that corresponds to mapped alteration is $\sim 1.8$ $\mathrm{km}^{3}$ (Finn and others, 2007), in the mid-range of the estimate based on geologic mapping. Moderately altered and fracturecontrolled areas of intense alteration were not detected with the geophysical mapping. The extensive area and volume of alteration estimated from geologic and geophysical mapping indicates that there has been a significant hydrothermal system in the past. Oxidation of hydrogen sulfide produced elemental sulfur and sulfuric acid that interacted with andesite to produce kaolinite, alunite, gypsum, and silica. Today, the most abundant sulfur-containing minerals are sulfates with some elemental sulfur and sparse pyrite (Hildreth and Fierstein, 1995).

Previous work did not identify thermal springs on Mount Adams (Cline, 1976; Fretwell, 1979), and the purpose of our work was to sample springs on Mount Adams to see if there were any slightly thermal springs. "Slightly thermal springs" are springs that do not meet the numerical criterion of Reed (1983) of being $10^{\circ} \mathrm{C}$ above the mean annual air temperature but have temperatures greater than nonthermal springs in the area. They usually also have dissolved constituents normally found in thermal waters (Nathenson and others, 2003). No slightly thermal springs were found in this study. Isotopic 
data for spring samples make it possible to develop isotopeelevation relations to estimate recharge elevations. Springs identified on topographic maps were visited to make physical measurements (table 1 and fig. 1). Some springs have high values of dissolved sulfate (see below), and they are shown with a different symbol. In addition, streams were measured to look for anomalies in temperature and (or) specific conductance. Flow rates were measured using a tape measure, float, and stop watch and are quite approximate. Locations for data taken in 1974 (Cline, 1976; Fretwell, 1979) are also shown on figure 1.

No fumaroles have been identified on Mount Adams. Hildreth and others (1983) smelled hydrogen sulfide in the summit area during field work in 1981 and 1982, but no specific orifices were identified. Earlier reports of summit fumaroles are summarized in Hildreth and others (1983). A gas vent was identified and sampled for this study.

\section{Characteristics of Springs and Streams}

\section{Air and Spring Temperatures}

In order to evaluate the temperature data for springs, we plot data for mean annual air temperatures from weather stations (National Oceanic and Atmospheric Administration, 1997a,b; Western Regional Climate Center, 2011a,b) within a block centered on Mount Adams (fig. 2) versus station elevation (fig. 3). The mean annual air temperatures are the "normal" values for the period 1961 to 1990. Air temperatures are mostly determined by station elevation (fig. 3), with some variability about this relation. Using least-squares correlation with air temperature as the dependent variable, the slope of the correlation line shown in figure 3 is $-0.00512 \pm 0.00036$ ${ }^{\circ} \mathrm{C} / \mathrm{m}$ and the intercept at sea level is $12.0 \pm 0.2{ }^{\circ} \mathrm{C}$ (standard errors of the mean). The data in figure 3 group into values warmer than the line and those cooler than the line. Many of the stations with warmer temperatures are along the eastern part of the area, and some are along the Columbia River at low elevations. There is no clear pattern that would indicate that data from some stations should not be included in calculating the correlation, and we accept the correlation line.

Spring temperatures measured in this study are generally cooler than air temperatures, though a few coincide with the correlation line (fig. 3). Four springs have data from 1974 and this study (color coded in figure 3), and their temperatures from the two samplings are generally pretty close in value. Two springs with data from 1974 are warmer than the correlation line for air temperature with elevation. Spring $07 / 12 \mathrm{E}-04 \mathrm{M} 01 \mathrm{~S}$ has a temperature of $8.0^{\circ} \mathrm{C}$; however, the values for sulfate and chloride concentrations are not anomalous (table 2). Spring 09/12E-35R01S has a temperature of $10.2^{\circ} \mathrm{C}$, specific conductance of 102 microsiemens per centimeter $(\mu \mathrm{S} / \mathrm{cm})$, and flow of 0.2 liters per second $(\mathrm{L} / \mathrm{s})$. Unfortunately, no chemical data are available for this spring. Both the specific conductance and temperature are high compared to other springs (except for those with high sulfate). If this spring is truly anomalous, its low flow makes it a rather small feature. In summary, all springs analyzed and discussed in this report are nonthermal.

\section{Chemistry of Springs and Streams}

The chemistry of most springs on Mount Adams is determined by the process of weathering of volcanic rock (Nathenson and Thompson, 1990). Carbon dioxide in the soil zone is much higher in partial pressure than in the atmosphere, and this carbon dioxide dissolves in groundwater, making an acid solution that reacts with minerals in volcanic rock. The result is bicarbonate, sodium, calcium, magnesium, and potassium ions going into solution along with silica as a neutral species, with the original minerals converting to clays. For example, a plot of sodium versus bicarbonate (fig. 4) shows that for most springs increasing bicarbonate concentrations result in increasing sodium concentrations following a linear relationship.

In addition to the products from weathering, water is some springs and streams contains added sulfate from either dissolving or reacting with hydrothermal minerals. Small amounts of sulfate and chloride are present in precipitation and become more concentrated from evaporation during snow melt or stream flow before water enters the ground. Springs have chloride content ranging as high as 0.8 milligrams per liter ( $\mathrm{mg} / \mathrm{L})$ (table 2) - about what one would expect from that in precipitation after some evaporative concentration. The lack of any chloride anomalies is consistent with most springs having temperatures that do not indicate any component of thermal water (fig. 3). Sulfate concentrations in some springs and creeks are elevated compared to most springs (table 2), and we have used this to identify a group of high-sulfate springs. Trappers Creek and Divide Camp Springs may also belong to this group, but we chose the clearly anomalous sulfate concentration of $3.5 \mathrm{mg} / \mathrm{L}$ in McCumber Spring as minimum value for inclusion in the group. The sodium concentrations for McCumber, Goat Butte, and Salt Creek springs are close to the sodium/bicarbonate correlation line (fig. 4), whereas the Salt Creek Spring no. 2 clearly has significantly more sodium than expected for its bicarbonate concentration. McCumber Spring in 1974 did not contain anomalous sulfate (table 2). The samples for the Klickitat River and Big Muddy Creek are essentially along the correlation line of figure 4 . Adams Creek and Cascade Creek have no bicarbonate, and their sodium and sulfate concentrations are from hydrothermal minerals.

Another way to see the differences between weathering and the addition of constituents from hydrothermal minerals is a plot of silica content versus specific conductance (fig. 5). In general, specific conductance of a spring's water is proportional to its bicarbonate concentration, but for springs with high sulfate content, it is proportional to the combination of bicarbonate and sulfate. For most springs in figure 5, silica concentrations are proportional to specific conductance. 
Table 1. Physical measurements and sampling information on springs and streams on and near Mount Adams.

[Measurements on springs at the orifice are identified by an * after the name. Cond. is specific conductance. Locations use North American Datum of 1927.]

\begin{tabular}{|c|c|c|c|c|c|c|c|c|c|c|}
\hline \multirow{2}{*}{ Loc. No. } & \multirow{2}{*}{ Name } & \multirow{2}{*}{ Sample No. } & \multicolumn{2}{|c|}{ Measurement point } & \multirow{2}{*}{ Elev. (m) } & \multirow{2}{*}{ Date } & \multirow{2}{*}{ Flow (L/s) } & \multirow{2}{*}{$\mathbf{T}\left({ }^{\circ} \mathbf{C}\right)$} & \multirow{2}{*}{ Cond. $(\mu \mathrm{S} / \mathrm{cm})$} & \multirow{2}{*}{ Field pH } \\
\hline & & & Latitude & Longitude & & & & & & \\
\hline $\mathrm{C} 03-01$ & Mile 38 Spring* & MNA-03-01 & $46^{\circ} 06.97^{\prime}$ & $121^{\circ} 36.60^{\prime}$ & 1,019 & $8 / 20 / 03$ & 0.33 & 5.3 & 33.3 & 5 \\
\hline $\mathrm{C} 03-02$ & Rock Spring* & MNA03-02 & $46^{\circ} 07.66^{\prime}$ & $121^{\circ} 33.38^{\prime}$ & 1,403 & $8 / 20 / 03$ & 0.10 & 2.4 & 30.8 & 4.7 \\
\hline $\mathrm{C} 03-03$ & Cascade Creek & & $46^{\circ} 06.34^{\prime}$ & $121^{\circ} 36.28^{\prime}$ & 935 & $8 / 20 / 03$ & 360 & 11.5 & 99.0 & \\
\hline $\mathrm{C} 03-04$ & White Salmon River & & $46^{\circ} 06.32^{\prime}$ & $121^{\circ} 36.48^{\prime}$ & 972 & $8 / 20 / 03$ & 360 & 8.9 & 53.0 & \\
\hline $\mathrm{C} 03-05$ & Morrison Creek & & $46^{\circ} 09.19^{\prime}$ & $121^{\circ} 29.80^{\prime}$ & 1,910 & $8 / 21 / 03$ & 24 & 8.7 & 1.2 & \\
\hline $\mathrm{C} 03-06$ & Stream & & $46^{\circ} 09.67^{\prime}$ & $121^{\circ} 30.48^{\prime}$ & 1,868 & $8 / 21 / 03$ & 5.4 & 8.7 & 42.3 & \\
\hline $\mathrm{C} 03-07$ & Stream & & $46^{\circ} 09.69$ & $121^{\circ} 30.69^{\prime}$ & 1,849 & $8 / 21 / 03$ & 22.5 & 7.4 & 42.3 & \\
\hline $\mathrm{C} 03-08$ & Stream & & $46^{\circ} 09.81^{\prime}$ & $121^{\circ} 31.07^{\prime}$ & 1,866 & $8 / 21 / 03$ & 2.7 & 12.3 & 66.5 & \\
\hline C03-09 & Salt Creek & & $46^{\circ} 09.91^{\prime}$ & $121^{\circ} 31.39^{\prime}$ & 1,865 & $8 / 21 / 03$ & 72 & 12.4 & 21.7 & \\
\hline $\mathrm{C} 03-10$ & Salt Creek Spring* & MNA03-03 & $46^{\circ} 09.86^{\prime}$ & $121^{\circ} 31.37^{\prime}$ & 1,869 & $8 / 21 / 03$ & 9.0 & 2.5 & 63.5 & 5 \\
\hline $\mathrm{C} 03-17$ & Aiken Lava Bed Spring* & MNA03-04 & $46^{\circ} 05.69^{\prime}$ & $121^{\circ} 29.07^{\prime}$ & 1,156 & $8 / 23 / 03$ & 24 & 2.5 & 15.4 & 5.5 \\
\hline $\mathrm{C} 03-18$ & Boundary Spring* & MNA03-05 & $46^{\circ} 09.07^{\prime}$ & $121^{\circ} 27.66^{\prime}$ & 1,962 & $8 / 24 / 03$ & 1.2 & 2.0 & 9.5 & 5 \\
\hline C03-19 & Campground Spring* & MNA03-06 & $46^{\circ} 08.14^{\prime}$ & $121^{\circ} 29.48^{\prime}$ & 1,691 & $8 / 24 / 03$ & 0.22 & 2.2 & 23.2 & 5 \\
\hline $\mathrm{C} 03-20$ & Divide Camp Spring* & MNA03-07 & $46^{\circ} 14.65^{\prime}$ & $121^{\circ} 33.43^{\prime}$ & 1,708 & $8 / 25 / 03$ & 0.25 & 1.2 & 21.0 & 5.5 \\
\hline $\mathrm{C} 03-21$ & Adams Creek & & $46^{\circ} 15.14^{\prime}$ & $121^{\circ} 33.38^{\prime}$ & 1,658 & $8 / 25 / 03$ & 360 & 8.3 & 136 & \\
\hline $\mathrm{C} 03-22$ & East Fork Adams Creek & & $46^{\circ} 16.41^{\prime}$ & $121^{\circ} 33.74^{\prime}$ & 1,392 & $8 / 25 / 03$ & 180 & 6.0 & 30.0 & \\
\hline $\mathrm{C} 03-23$ & Killen Creek & & $46^{\circ} 17.63^{\prime}$ & $121^{\circ} 32.83^{\prime}$ & 1,391 & $8 / 25 / 03$ & 60 & 11.3 & 17.0 & \\
\hline $\mathrm{C} 03-24$ & South Fork Spring Creek & & $46^{\circ} 18.40^{\prime}$ & $121^{\circ} 32.67^{\prime}$ & 1,348 & $8 / 25 / 03$ & 150 & 8.6 & 35.0 & \\
\hline $\mathrm{C} 03-25$ & Muddy Fork & & $46^{\circ} 19.28^{\prime}$ & $121^{\circ} 32.69^{\prime}$ & 1,299 & $8 / 25 / 03$ & 480 & 11.7 & 30.0 & \\
\hline $\mathrm{C} 03-26$ & Outflow from Salt Creek Springs & & $46^{\circ} 09.86^{\prime}$ & $121^{\circ} 31.37^{\prime}$ & 1,869 & $8 / 26 / 03$ & 18 & 2.6 & 65.0 & \\
\hline $\mathrm{C} 03-27$ & Salt Creek Spring No. $2^{*}$ & MNA03-08 & $46^{\circ} 09.93^{\prime}$ & $121^{\circ} 31.55^{\prime}$ & 1,856 & $8 / 26 / 03$ & 150 & 1.6 & 114.2 & 5.5 \\
\hline $\mathrm{C} 03-28$ & Stream & & $46^{\circ} 10.02^{\prime}$ & $121^{\circ} 31.67^{\prime}$ & 1,858 & $8 / 26 / 03$ & 78 & 13.0 & 113.4 & \\
\hline $\mathrm{C} 03-29$ & Cascade Creek & & $46^{\circ} 10.54^{\prime}$ & $121^{\circ} 32.65$ & 1,849 & $8 / 26 / 03$ & 30 & 14.6 & 87.2 & \\
\hline $\mathrm{C} 03-30$ & Cascade Creek & MNA03-09 & $46^{\circ} 10.65^{\prime}$ & $121^{\circ} 32.84^{\prime}$ & 1,843 & $8 / 26 / 03$ & 180 & 9.8 & 224 & 4 \\
\hline $\mathrm{C} 03-31$ & Wicky Creek Spring* & MNA03-10 & $46^{\circ} 05.88^{\prime}$ & $121^{\circ} 31.77^{\prime}$ & 1,158 & $8 / 27 / 03$ & 6 & 4.0 & 55.8 & 5.5 \\
\hline $\mathrm{C} 03-32$ & Creek & & $46^{\circ} 11.87^{\prime}$ & $121^{\circ} 38.61^{\prime}$ & 1,172 & $8 / 27 / 03$ & 200 & 4.6 & 33.6 & \\
\hline $\mathrm{C} 03-33$ & Twin Falls Creek & & $46^{\circ} 12.68^{\prime}$ & $121^{\circ} 37.83^{\prime}$ & 1,178 & $8 / 27 / 03$ & 120 & 3.7 & 59.7 & \\
\hline $\mathrm{C} 03-34$ & Noname Creek & & $46^{\circ} 13.21^{\prime}$ & $121^{\circ} 37.72^{\prime}$ & 1,106 & $8 / 27 / 03$ & 25 & 6.4 & 96.4 & \\
\hline $\mathrm{C} 03-35$ & Riley Creek & & $46^{\circ} 13.67^{\prime}$ & $121^{\circ} 37.21^{\prime}$ & 1,096 & $8 / 27 / 03$ & 480 & 7.8 & 35.0 & \\
\hline $\mathrm{C} 03-36$ & Big Spring Creek & & $46^{\circ} 13.93^{\prime}$ & $121^{\circ} 37.44$ & 1,096 & $8 / 27 / 03$ & 540 & 4.2 & 31.5 & \\
\hline $\mathrm{C} 03-37$ & Lewis River & & $46^{\circ} 15.30^{\prime}$ & $121^{\circ} 36.45^{\prime}$ & 1,205 & $8 / 27 / 03$ & 240 & 9.1 & 47.8 & \\
\hline C04-01 & McCumber Spring* & MNA04-01 & $46^{\circ} 04.31^{\prime}$ & $121^{\circ} 18.30^{\prime}$ & 750 & $8 / 25 / 04$ & 38 & 5.5 & 52.0 & 5.5 \\
\hline $\mathrm{C} 04-02$ & Bup Spring* & MNA04-02 & $46^{\circ} 11.06^{\prime}$ & $121^{\circ} 17.32^{\prime}$ & 818 & $8 / 25 / 04$ & 0.7 & 5.6 & 69.5 & 5.5 \\
\hline
\end{tabular}


Table 1. Physical measurements and sampling information on springs and streams on and near Mount Adams.-Continued

\begin{tabular}{|c|c|c|c|c|c|c|c|c|c|c|}
\hline \multirow{2}{*}{ Loc. No. } & \multirow{2}{*}{ Name } & \multirow{2}{*}{ Sample No. } & \multicolumn{2}{|c|}{ Measurement point } & \multirow{2}{*}{ Elev. (m) } & \multirow{2}{*}{ Date } & \multirow{2}{*}{ Flow (L/s) } & \multirow{2}{*}{$\mathbf{T}\left({ }^{\circ} \mathrm{C}\right)$} & \multirow{2}{*}{ Cond. $(\mu \mathrm{S} / \mathrm{cm})$} & \multirow{2}{*}{ Field pH } \\
\hline & & & Latitude & Longitude & & & & & & \\
\hline $\mathrm{C} 04-03$ & Clearwater Creek & & $46^{\circ} 18.52^{\prime}$ & $121^{\circ} 24.42^{\prime}$ & 1,292 & $8 / 26 / 04$ & 230 & 4.2 & 43.0 & \\
\hline $\mathrm{C} 04-04$ & Cold Creek & & $46^{\circ} 18.83^{\prime}$ & $121^{\circ} 22.78^{\prime}$ & 1,185 & $8 / 26 / 04$ & 72 & 5.8 & 81.5 & \\
\hline $\mathrm{C} 04-05$ & Trappers Creek Spring* & MNA04-03 & $46^{\circ} 16.70^{\prime}$ & $121^{\circ} 24.12^{\prime}$ & 1,409 & $8 / 26 / 04$ & 450 & 3.2 & 53.3 & 5.5 \\
\hline C04-06 & Trappers Creek & & $46^{\circ} 16.85^{\prime}$ & $121^{\circ} 23.26^{\prime}$ & 1,320 & $8 / 26 / 04$ & 540 & 4.6 & 53.3 & \\
\hline $\mathrm{C} 04-07$ & Little Muddy Creek & & $46^{\circ} 16.21^{\prime}$ & $121^{\circ} 22.73^{\prime}$ & 1,351 & $8 / 26 / 04$ & 360 & 9.5 & 40.6 & \\
\hline $\mathrm{C} 04-08$ & Bird Creek Meadows stream & & $46^{\circ} 09.13^{\prime}$ & $121^{\circ} 26.09^{\prime}$ & 1,828 & $8 / 27 / 04$ & 18 & 7.7 & 13.1 & \\
\hline C04-09 & Stream & & $46^{\circ} 09.07^{\prime}$ & $121^{\circ} 26.34^{\prime}$ & 1,853 & $8 / 27 / 04$ & 23 & 6.2 & 14.3 & \\
\hline $\mathrm{C} 04-10$ & Stream & & $46^{\circ} 08.99^{\prime}$ & $121^{\circ} 26.58^{\prime}$ & 1,873 & $8 / 27 / 04$ & 36 & 4.4 & 13.5 & \\
\hline $\mathrm{C} 04-11$ & Stream & & $46^{\circ} 08.96^{\prime}$ & $121^{\circ} 26.69^{\prime}$ & 1,867 & $8 / 27 / 04$ & 80 & 8.4 & 7.9 & \\
\hline $\mathrm{C} 04-12$ & Spring* & & $46^{\circ} 08.93^{\prime}$ & $121^{\circ} 26.88^{\prime}$ & 1,871 & $8 / 27 / 04$ & 5.4 & 1.7 & 12.0 & \\
\hline $\mathrm{C} 04-13$ & Stream & & $46^{\circ} 08.94^{\prime}$ & $121^{\circ} 26.95^{\prime}$ & 1,878 & $8 / 27 / 04$ & 60 & 6.6 & 6.6 & \\
\hline $\mathrm{C} 04-14$ & Stream & & $46^{\circ} 08.96^{\prime}$ & $121^{\circ} 27.11^{\prime}$ & 1,892 & $8 / 27 / 04$ & 56 & 3.5 & 8.4 & \\
\hline $\mathrm{C} 04-15$ & Crooked Creek & & $46^{\circ} 08.99^{\prime}$ & $121^{\circ} 27.27^{\prime}$ & 1,889 & $8 / 27 / 04$ & 45 & 4.9 & 9.2 & \\
\hline C04-16 & Crooked Creek Spring* & MNA04-04 & $46^{\circ} 09.33^{\prime}$ & $121^{\circ} 27.53^{\prime}$ & 2,010 & $8 / 27 / 04$ & 54 & 0.8 & 9.6 & 5 \\
\hline $\mathrm{C} 04-17$ & Meadow Spring* & MNA04-05 & $46^{\circ} 09.45^{\prime}$ & $121^{\circ} 25.59^{\prime}$ & 1,637 & $8 / 28 / 04$ & 60 & 2.6 & 22.8 & 5.5 \\
\hline C04-18 & Hellroaring Creek & & $46^{\circ} 09.82^{\prime}$ & $121^{\circ} 23.99^{\prime}$ & 1,457 & $8 / 28 / 04$ & 360 & 8.3 & 17.6 & \\
\hline C04-19 & Island Spring* & MNA04-06 & $46^{\circ} 10.09^{\prime}$ & $121^{\circ} 23.81^{\prime}$ & 1,604 & $8 / 28 / 04$ & 1.8 & 3.8 & 21.2 & 5 \\
\hline $\mathrm{C} 04-20$ & Spring stream & & $46^{\circ} 06.18^{\prime}$ & $121^{\circ} 23.74^{\prime}$ & 1,281 & $8 / 29 / 04$ & -- & 10.6 & 53.3 & \\
\hline $\mathrm{C} 04-21$ & Bacon Creek Spring* & MNA04-07 & $46^{\circ} 07.16^{\prime}$ & $121^{\circ} 22.05^{\prime}$ & 1,260 & $8 / 29 / 04$ & 18 & 2.9 & 40.2 & 5 \\
\hline $\mathrm{C} 04-22$ & Cress Camp Spring* & & $46^{\circ} 08.21^{\prime}$ & $121^{\circ} 21.73^{\prime}$ & 1,393 & $8 / 29 / 04$ & 7.2 & 3.0 & 30.3 & \\
\hline $\mathrm{C} 04-23$ & Soda Spring Creek & & $46^{\circ} 13.77^{\prime}$ & $121^{\circ} 17.45^{\prime}$ & 1,052 & $8 / 30 / 04$ & 6.0 & 11.7 & 79.8 & \\
\hline $\mathrm{C} 04-24$ & Spring & & $46^{\circ} 13.65^{\prime}$ & $121^{\circ} 22.08^{\prime}$ & 1,473 & $8 / 30 / 04$ & dry & & & \\
\hline $\mathrm{C} 04-25$ & Cunningham Creek & & $46^{\circ} 10.86^{\prime}$ & $121^{\circ} 17.18^{\prime}$ & 819 & $8 / 30 / 04$ & 180 & 10.7 & 50.5 & \\
\hline $\mathrm{C} 04-26$ & Big Muddy Creek & & $46^{\circ} 09.10^{\prime}$ & $121^{\circ} 17.58^{\prime}$ & 742 & $8 / 30 / 04$ & 2,500 & 12.5 & 65.8 & \\
\hline $\mathrm{C} 04-27$ & Cougar Creek & & $46^{\circ} 08.32^{\prime}$ & $121^{\circ} 17.53^{\prime}$ & 789 & $8 / 30 / 04$ & 9.0 & 13.5 & 49.0 & \\
\hline $\mathrm{C} 04-28$ & Dairy Creek & & $46^{\circ} 06.30^{\prime}$ & $121^{\circ} 17.78^{\prime}$ & 699 & $8 / 30 / 04$ & 18 & 12.4 & 78.0 & \\
\hline C04-29 & Bacon Creek & & $46^{\circ} 04.71^{\prime}$ & $121^{\circ} 17.17^{\prime}$ & 669 & $8 / 30 / 04$ & 12 & 10.9 & 55.0 & \\
\hline $\mathrm{C} 04-30$ & Bird Creek & & $46^{\circ} 03.59^{\prime}$ & $121^{\circ} 18.28^{\prime}$ & 678 & $8 / 30 / 04$ & 90 & 12.3 & 24.6 & \\
\hline $\mathrm{C} 04-31$ & Adams Creek & MNA04-08 & $46^{\circ} 14.48^{\prime}$ & $121^{\circ} 32.62^{\prime}$ & 1,825 & $8 / 31 / 04$ & 900 & 6.2 & 101.9 & 4.5 \\
\hline $\mathrm{C} 04-32$ & Adams Creek & & $46^{\circ} 14.49^{\prime}$ & $121^{\circ} 32.58^{\prime}$ & 1,839 & $8 / 31 / 04$ & 36 & 9.9 & 88.2 & \\
\hline $\mathrm{C} 04-33$ & Lewis River & & $46^{\circ} 14.25^{\prime}$ & $121^{\circ} 32.83^{\prime}$ & 1,850 & $8 / 31 / 04$ & 270 & 9.6 & 62.6 & \\
\hline $\mathrm{C} 04-34$ & Stream & & $46^{\circ} 14.12^{\prime}$ & $121^{\circ} 33.01^{\prime}$ & 1,849 & $8 / 31 / 04$ & 4.8 & 19.7 & 15.5 & \\
\hline $\mathrm{C} 04-35$ & Mutton Creek & & $46^{\circ} 13.75^{\prime}$ & $121^{\circ} 33.39^{\prime}$ & 1,861 & $8 / 31 / 04$ & dry & & & \\
\hline $\mathrm{C} 04-36$ & Goat Butte Spring* & MNA04-09 & $46^{\circ} 12.46^{\prime}$ & $121^{\circ} 26.10^{\prime}$ & 2,012 & $9 / 1 / 04$ & 12 & 1.2 & 32.6 & 5 \\
\hline
\end{tabular}




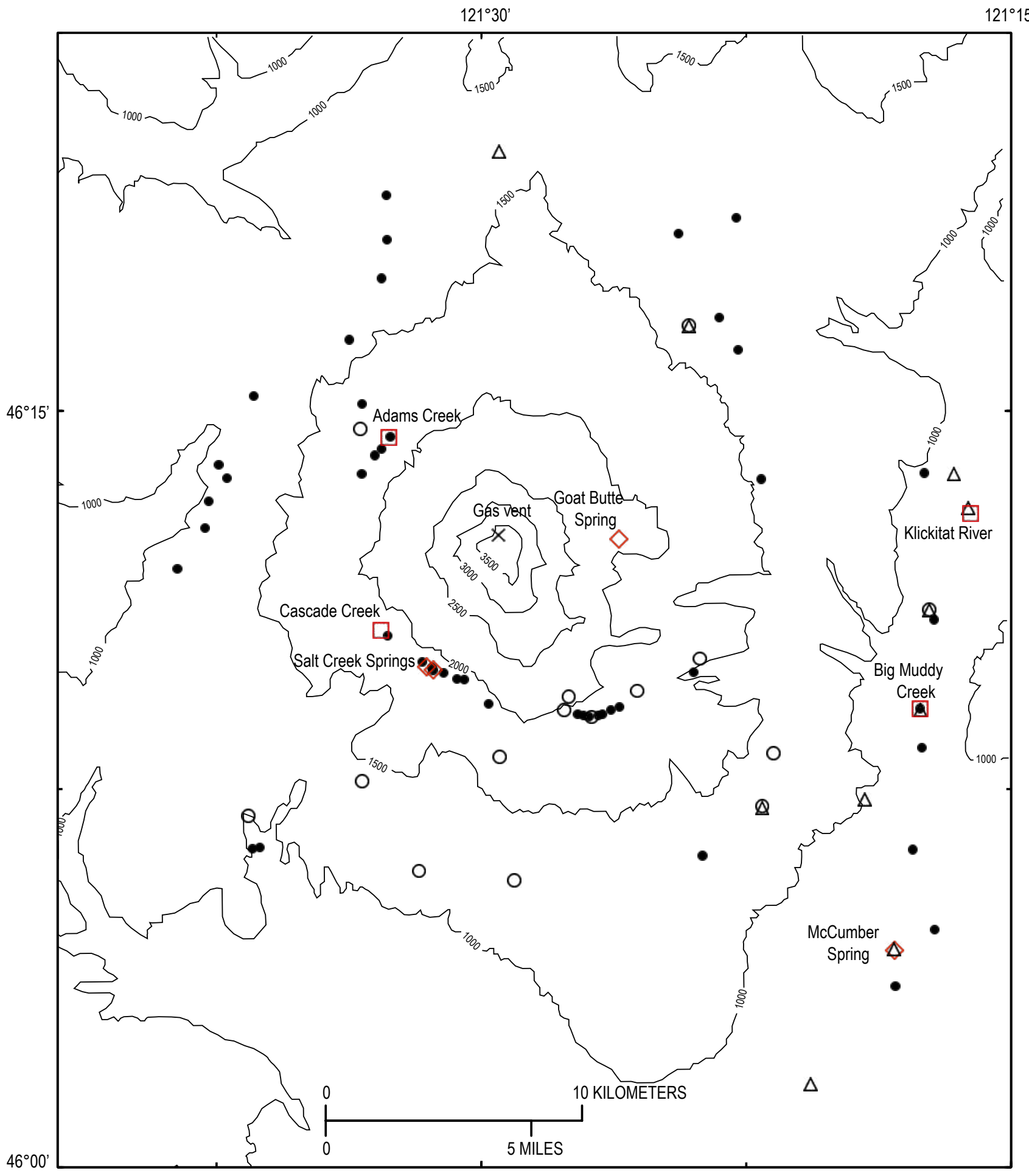

EXPLANATION
- Measured streams
$\square$ High-sulfate streams
OSprings
$\Delta$ Springs sampled in 1974
$\diamond$ High-sulfate springs
$\times$ Gas vent

Figure 1. Map of Mount Adams, Washington, showing locations of sites visited in this study and those from 1974 (Cline, 1976; Fretwell, 1979). High-sulfate springs have $\geq 3.5 \mathrm{mg} / \mathrm{L}$ sulfate. Topography (contours in meters) modified from Hildreth and Lanphere (1994). 


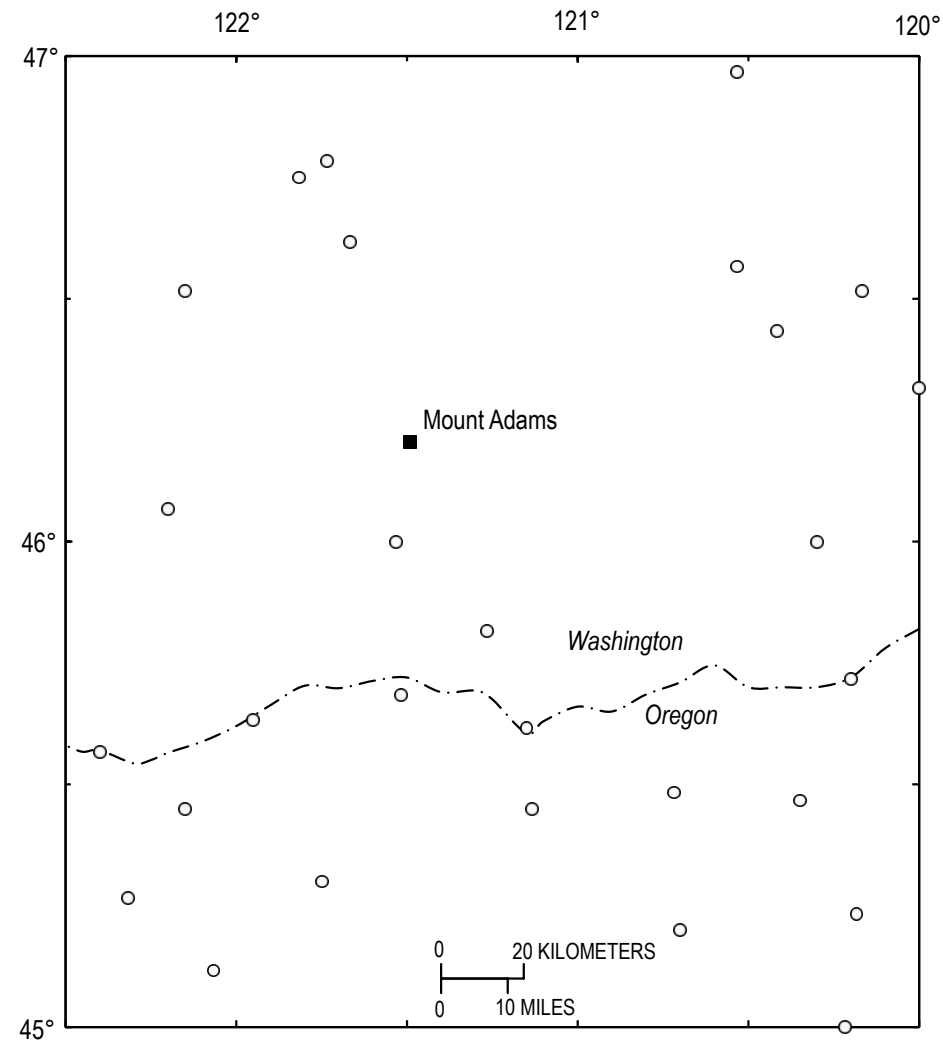

Figure 2. Map of weather stations in the vicinity of Mount Adams with 30-year records for calculation of normal air temperatures. Dash-dot line shows state boundary.

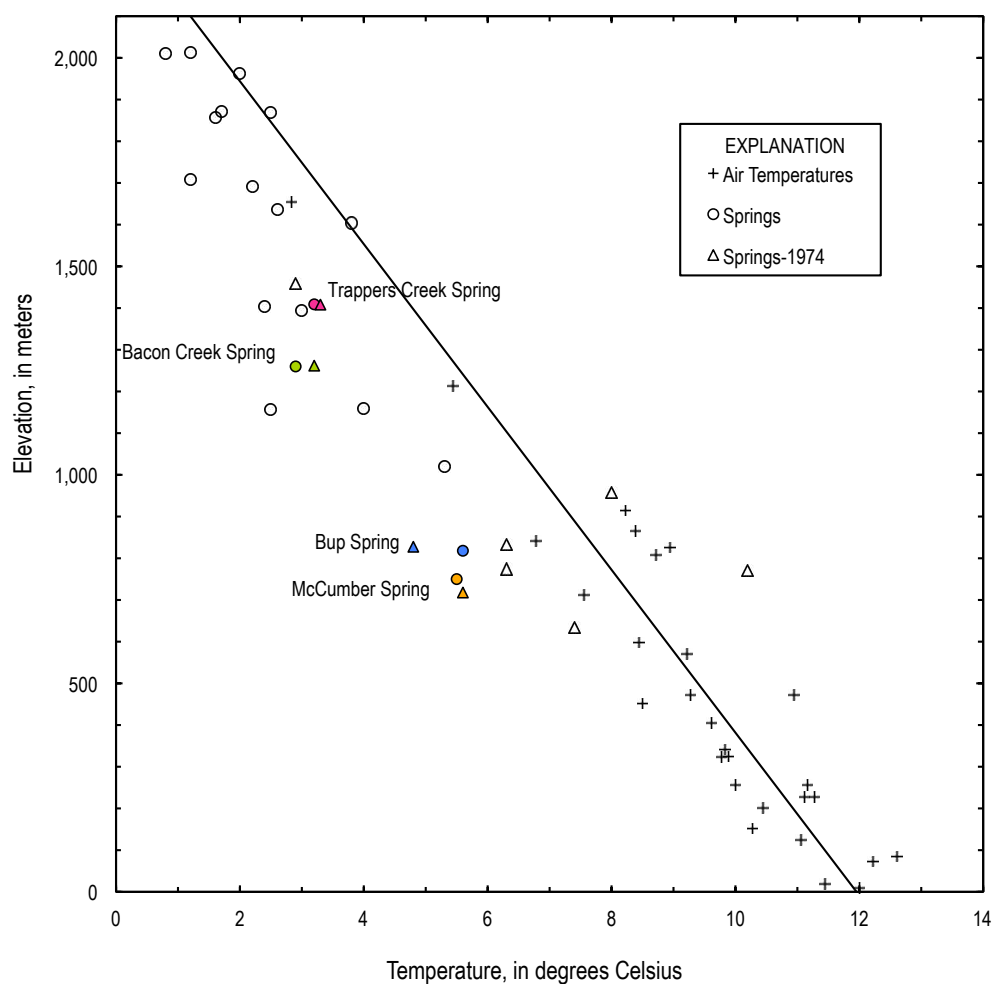

Figure 3. Graph of temperature versus elevation for air temperatures from weather stations shown in figure 2 and for temperatures of springs from sites in figure 1. Correlation line is for air temperatures. For springs with temperature measurements from both 1974 and this study, the two symbols are shown in the same color. 


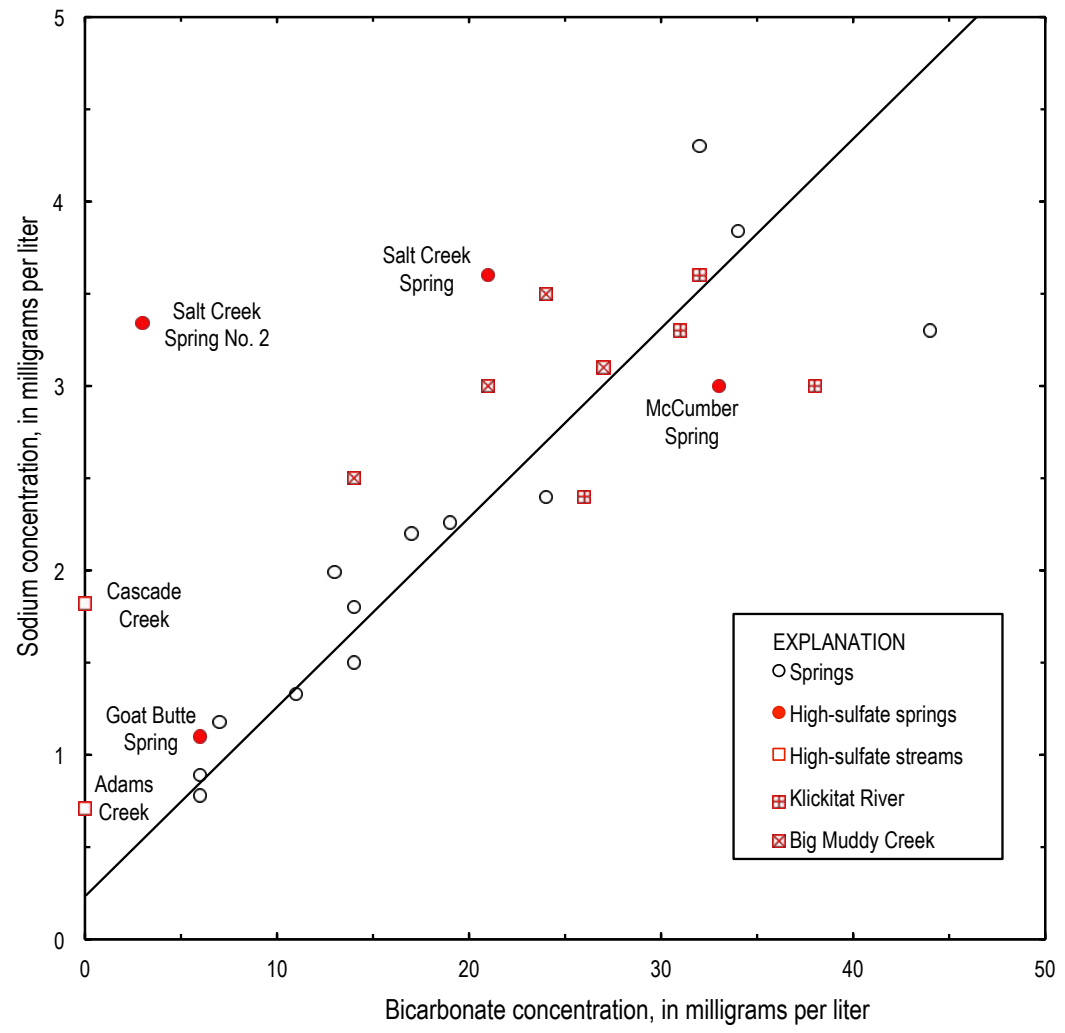

Figure 4. Graph of sodium versus bicarbonate concentrations for springs and streams in the Mount Adams region. Correlation line is for springs (circles), with bicarbonate as the dependent variable.

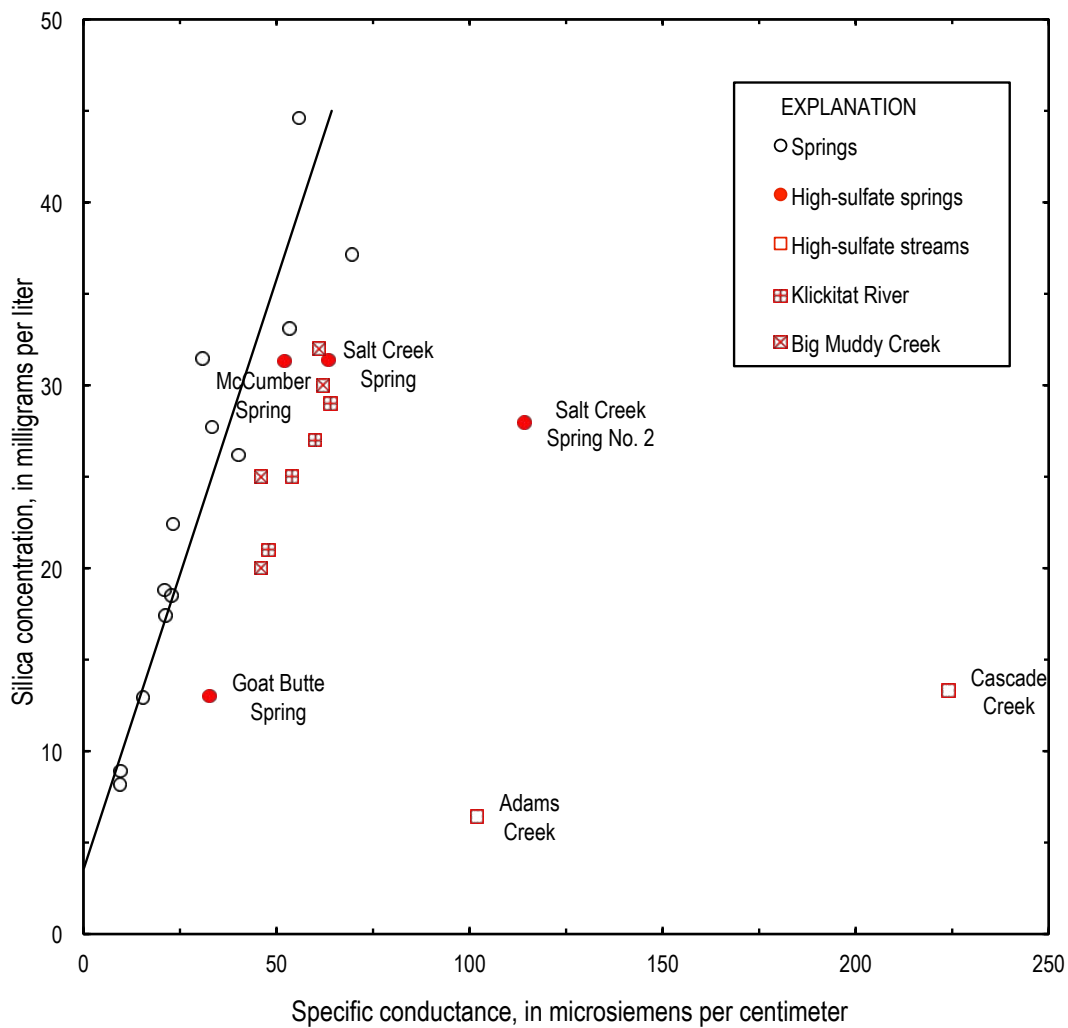

Figure 5. Graph of silica concentration versus specific conductance for springs and streams in the Mount Adams region. Correlation line is for springs (circles), with specific conductance as the dependent variable. 
Table 2. Chemical and isotopic data for water samples from springs and streams on and near Mount Adams.

[Measurements on springs at the orifice are identified by an * after the name. Data obtained in 1974 from Cline (1976) and Fretwell (1979). -- indicates not analyzed or measured. Cond. is specific conductance. Locations use North American Datum of 1927. Chemical analyses by Mark Huebner and W.C. Evans and isotopic analyses from USGS stable isotope laboratory, Reston, VA.]

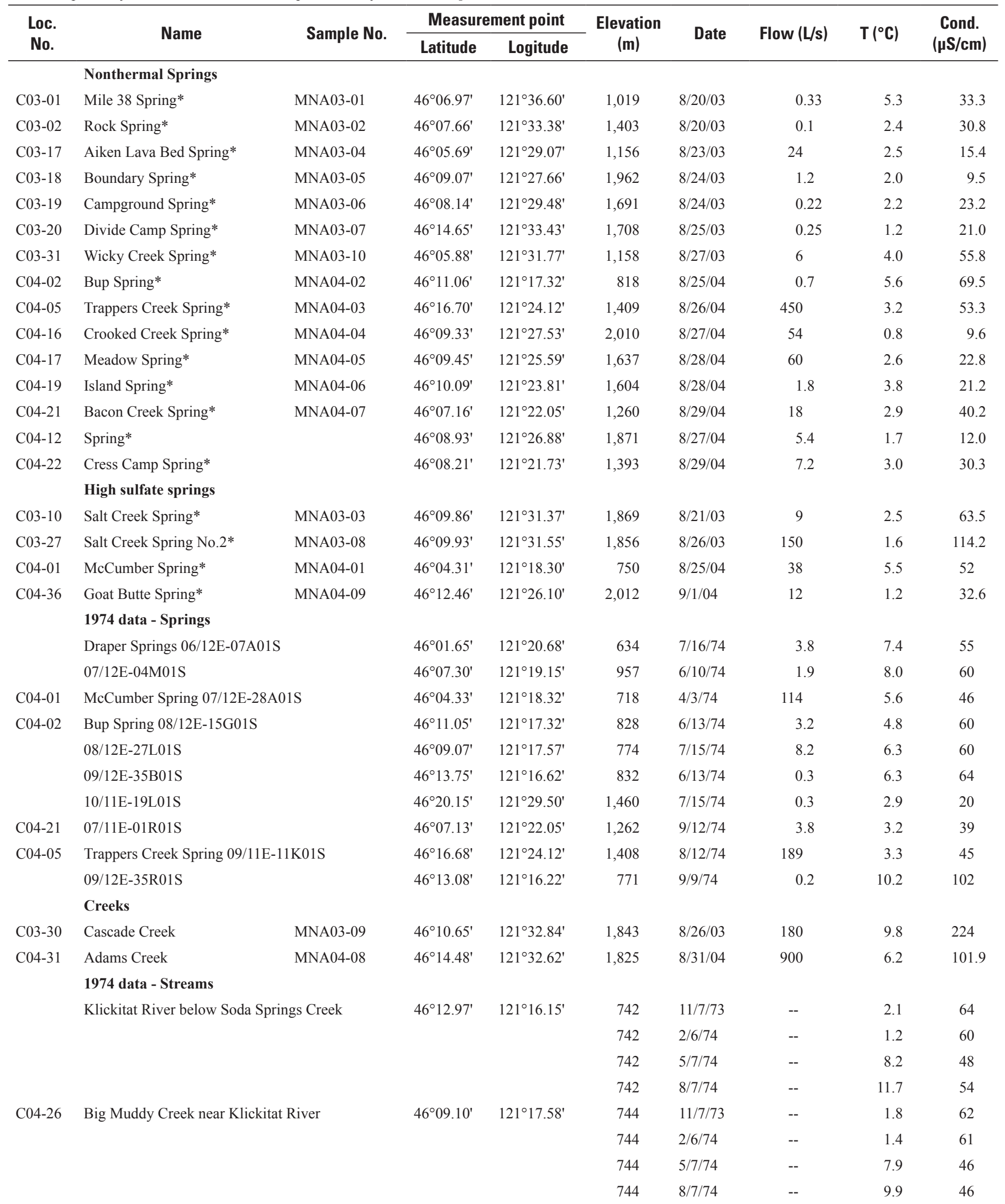


Table 2. Chemical and isotopic data for water samples from springs and streams on and near Mount Adams. - Continued

\begin{tabular}{|c|c|c|c|c|c|c|c|c|c|c|}
\hline $\begin{array}{c}\text { Field } \\
\mathrm{pH}\end{array}$ & $\mathrm{SiO}_{2}$ & Ca & $\mathrm{Mg}$ & $\mathrm{Na}$ & K & $\mathrm{HCO}_{3}$ & $\mathrm{SO}_{4}$ & $\begin{array}{c}\mathrm{CI} \\
\end{array}$ & $\begin{array}{c}\delta D \\
(\%)\end{array}$ & $\begin{array}{l}\delta^{18} 0 \\
(\%)\end{array}$ \\
\hline 4.7 & 31.5 & 1.82 & 0.56 & 2.2 & 1.69 & 17 & 0.2 & 0.6 & -94.5 & -13.41 \\
\hline 5.5 & 12.9 & 1.11 & 0.23 & 1.18 & 0.56 & 7 & 0.24 & 0.3 & -101.3 & -14.04 \\
\hline 5.5 & 18.8 & 1.57 & 0.44 & 1.33 & 0.68 & 11 & 1.07 & 0.37 & -96.8 & -13.55 \\
\hline 5.5 & 44.6 & 4.01 & 0.98 & 3.84 & 2.53 & 34 & 0.19 & 0.64 & -95.5 & -13.5 \\
\hline 5.5 & 37.1 & 6.7 & 2.9 & 3.3 & 1.4 & 44 & 0.2 & 0.4 & -102.0 & -14.0 \\
\hline 5.5 & 33.1 & 3.8 & 1.9 & 4.3 & 1.8 & 32 & 1.9 & 0.4 & -104.8 & -14.4 \\
\hline 5 & 26.2 & 3.7 & 1.2 & 2.4 & 1.3 & 24 & 0.12 & 0.3 & -100.8 & -13.9 \\
\hline-- & -- & -- & -- & -- & -- & -- & -- & -- & -- & -- \\
\hline-- & -- & -- & -- & -- & -- & -- & -- & -- & -- & -- \\
\hline 5 & 31.4 & 4.71 & 1.49 & 3.6 & 1.56 & 21 & 13 & 0.28 & -106.8 & -14.85 \\
\hline 5.5 & 28.0 & 9.84 & 2.2 & 3.34 & 0.81 & 3 & 43.9 & 0.29 & -102.9 & -14.39 \\
\hline 5.5 & 31.3 & 4.7 & 1.8 & 3 & 1.6 & 33 & 3.5 & 0.4 & -100.7 & -13.9 \\
\hline 5 & 13.0 & 3.3 & 0.7 & 1.1 & 0.81 & 6 & 8.9 & 0.16 & -115.8 & -15.6 \\
\hline-- & -- & 4.2 & 2.2 & 2.6 & 1.9 & 33 & 0.1 & 0.5 & -- & -- \\
\hline -- & -- & -- & -- & -- & -- & -- & -- & -- & -- & -- \\
\hline-- & -- & -- & -- & -- & -- & -- & -- & -- & -- & -- \\
\hline-- & -- & -- & -- & -- & -- & -- & -- & -- & -- & -- \\
\hline 4 & 13.3 & 10.21 & 2.95 & 1.82 & 0.49 & 0 & 72.3 & 0.2 & -- & -- \\
\hline 4.5 & 6.4 & 4.2 & 0.74 & 0.71 & 0.43 & 0 & 24 & 0.07 & -- & -- \\
\hline -- & 29 & 7.2 & 2.5 & 3.0 & 1.7 & 38 & 2.5 & 1.3 & -- & -- \\
\hline -- & 27 & 4.8 & 2.2 & 3.6 & 1.3 & 32 & 2.1 & 1.4 & -- & -- \\
\hline-- & 21 & 3.9 & 1.5 & 2.4 & 0.9 & 26 & 2.3 & 0.8 & -- & -- \\
\hline-- & 25 & 4.3 & 1.6 & 3.3 & 0.8 & 31 & 2.5 & 0.8 & -- & -- \\
\hline-- & 30 & 6.3 & 2.4 & 3.1 & 2.0 & 27 & 9.1 & 1.1 & -- & -- \\
\hline-- & 32 & 4.1 & 1.9 & 3.5 & 1.8 & 24 & 6.1 & 1.0 & -- & -- \\
\hline -- & 25 & 2.6 & 1.3 & 3.0 & 1.4 & 21 & 4.8 & 0.3 & -- & -- \\
\hline-- & 20 & 3.2 & 1.5 & 2.5 & 0.9 & 14 & 8.7 & 0.7 & -- & -- \\
\hline
\end{tabular}


Table 2. Chemical and isotopic data for water samples from springs and streams on and near Mount Adams. - Continued [Measurements on springs at the orifice are identified by an * after the name. Data obtained in 1974 from Cline (1976) and Fretwell (1979). -- indicates not analyzed or measured. Cond. is specific conductance. Locations use North American Datum of 1927. Chemical analyses by Mark Huebner and W.C. Evans and isotopic analyses from USGS stable isotope laboratory, Reston, VA.]

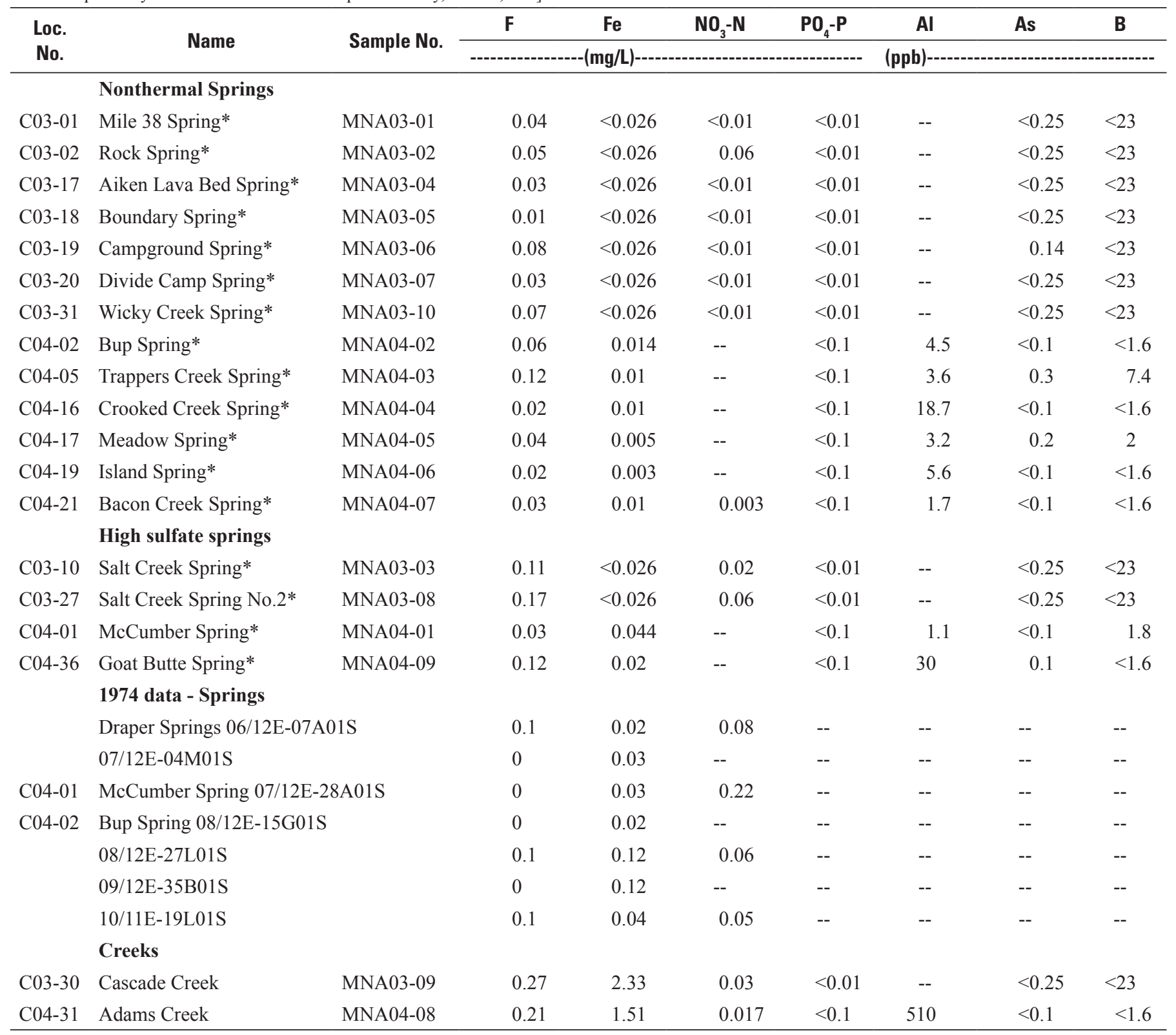


Table 2. Chemical and isotopic data for water samples from springs and streams on and near Mount Adams.-Continued

\begin{tabular}{|c|c|c|c|c|c|c|c|c|c|c|}
\hline $\mathrm{Ba}$ & $\mathrm{Cu}$ & Li & $\mathbf{M n}$ & Mo & $\mathrm{Pb}$ & $\mathbf{R b}$ & $\mathrm{Sr}$ & $\mathbf{U}$ & V & $\mathrm{Zn}$ \\
\hline 1.09 & -- & 0.38 & 0.3 & 0.03 & 0.009 & 4.88 & 19.45 & 0.005 & 0.74 & $<2.1$ \\
\hline 0.24 & -- & 0.7 & $<0.17$ & 0.08 & 0.055 & 7.1 & 15 & 0.01 & 0.32 & $<2.1$ \\
\hline 1.14 & -- & 1.13 & 0.07 & 0.03 & 0.191 & 3.34 & 10.12 & 0.003 & 1.83 & 1.22 \\
\hline 2.55 & -- & 0.15 & 0.08 & $<0.16$ & $<0.011$ & 1.7 & 9.28 & 0.001 & 0.39 & $<2.1$ \\
\hline 0.05 & -- & 1.61 & $<0.17$ & 0.19 & 0.035 & 4.71 & 8.31 & 0.008 & 6.11 & 2.49 \\
\hline 1.21 & -- & 0.35 & $<0.17$ & 0.04 & 0.05 & 2.3 & 13.07 & 0.005 & $<0.36$ & $<2.1$ \\
\hline 2.83 & -- & 2.1 & $<0.17$ & 0.2 & 0.035 & 9.71 & 58.23 & 0.025 & 5.28 & $<2.1$ \\
\hline 2.85 & 0.34 & 0.35 & $<0.1$ & 0.05 & 0.01 & 2.34 & 40 & 0.009 & 1.40 & 0.8 \\
\hline 1.56 & 0.63 & 5.5 & $<0.1$ & 0.46 & 0.1 & 6.03 & 18.3 & 0.064 & 6.4 & 2.2 \\
\hline 1.51 & 0.67 & 0.52 & 0.1 & 0.07 & 0.07 & 1.58 & 5.9 & $<0.004$ & 1.47 & 0.6 \\
\hline 1.41 & 1.17 & 1.59 & $<0.1$ & 0.15 & 0.07 & 2.67 & 12.7 & 0.005 & 0.55 & 0.6 \\
\hline 2.39 & 0.21 & 0.16 & $<0.1$ & 0.04 & 0.04 & 2.01 & 13.7 & $<0.004$ & 0.76 & 0.6 \\
\hline 0.41 & 1.03 & 0.54 & 1.21 & 0.10 & 0.1 & 4.1 & 16.4 & 0.009 & 1.43 & 0.7 \\
\hline 2.61 & -- & 3.39 & 0.28 & 0.26 & 0.065 & 6.35 & 31.43 & 0.029 & 3.93 & 6.14 \\
\hline 13.21 & -- & 2.85 & 54.6 & $<0.16$ & 0.023 & 3.66 & 40.09 & 0.022 & 0.65 & 3.72 \\
\hline 0.42 & 0.35 & 1.18 & 0.1 & 0.11 & $<0.01$ & 5.39 & 28 & 0.014 & 0.87 & 1.1 \\
\hline 0.84 & 1.01 & 0.84 & 0.32 & 0.09 & 0.06 & 2.29 & 9.3 & $<0.004$ & 2.57 & 1.0 \\
\hline-- & -- & -- & 0 & -- & -- & -- & -- & -- & -- & -- \\
\hline -- & -- & -- & 0 & -- & -- & -- & -- & -- & -- & -- \\
\hline -- & -- & -- & 0 & -- & -- & -- & -- & -- & -- & -- \\
\hline -- & -- & -- & 0 & -- & -- & -- & -- & -- & -- & -- \\
\hline -- & -- & -- & 0 & -- & -- & -- & -- & -- & -- & -- \\
\hline -- & -- & -- & 0 & -- & -- & -- & -- & -- & -- & -- \\
\hline -- & -- & -- & 0 & -- & -- & -- & -- & -- & -- & -- \\
\hline 1.34 & -- & 1.77 & 197 & $<0.16$ & 0.098 & 2.35 & 18.6 & 0.18 & $<0.36$ & 22.4 \\
\hline 1.75 & 18.9 & 0.76 & 55.5 & $<0.02$ & 0.04 & 0.94 & 6.6 & 0.101 & 0.10 & 9.9 \\
\hline
\end{tabular}




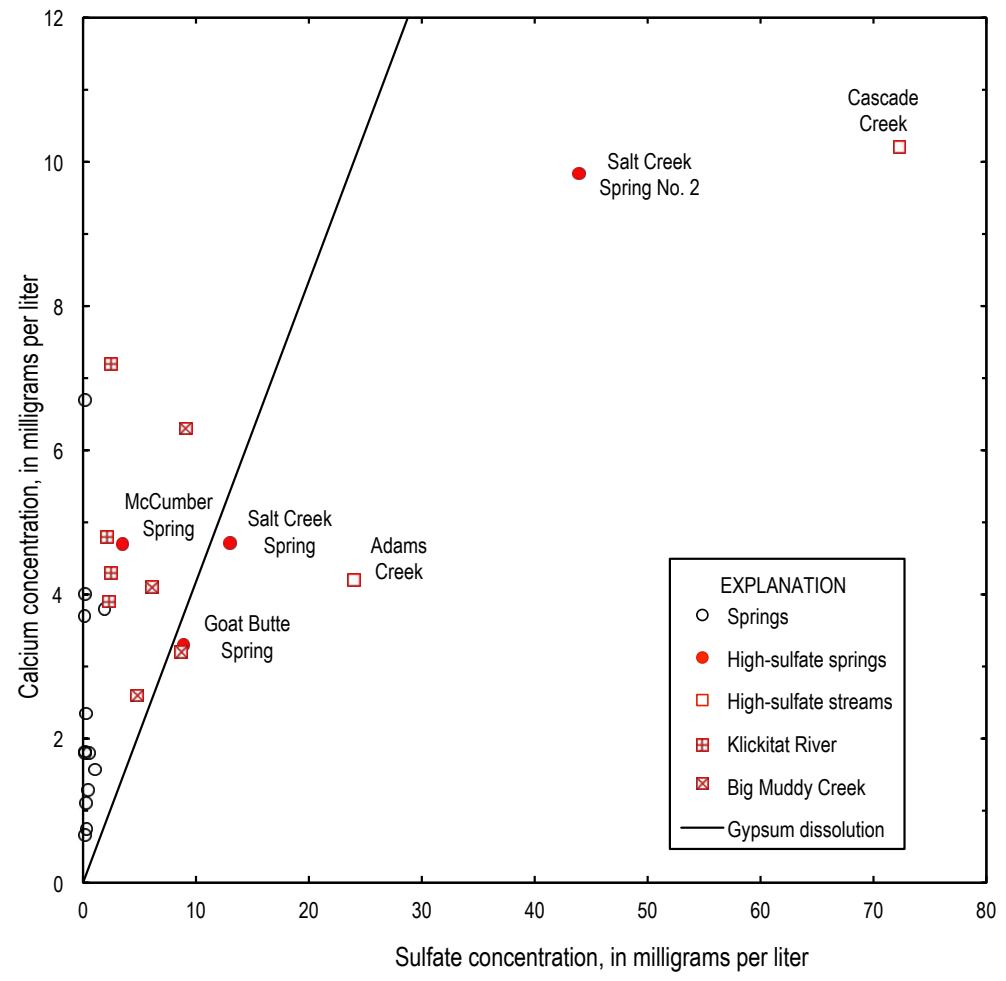

Figure 6. Graph of calcium versus sulfate concentrations for springs and streams in the Mount Adams region. Line is for the ratio of calcium to sulfate if gypsum is dissolved in water.

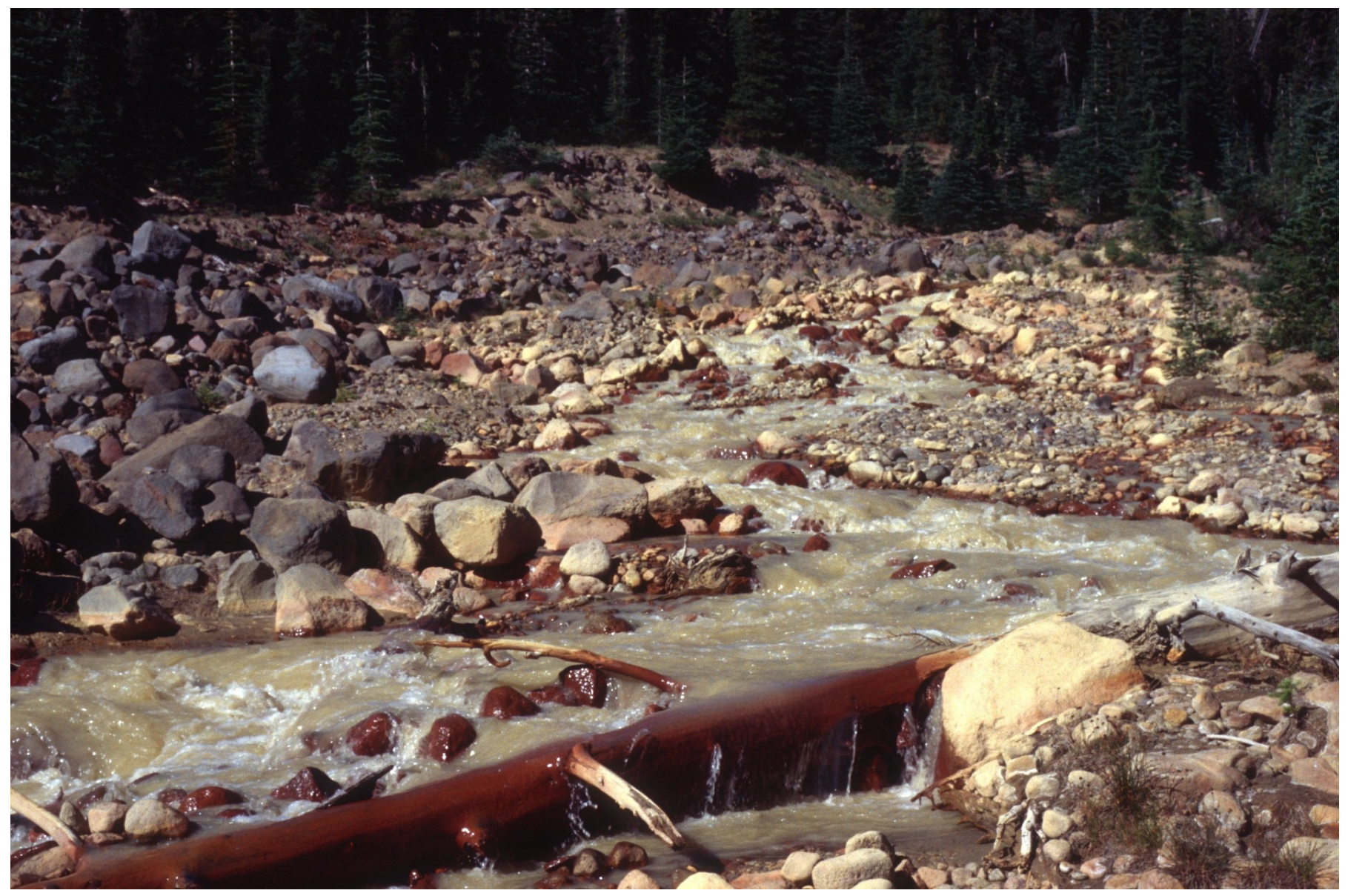

Figure 7. Photo of Cascade Creek at the location of sampling, showing the reddish-brown color of wetted precipitates. White boulder at lower right is about $1 / 2$ meter across. 


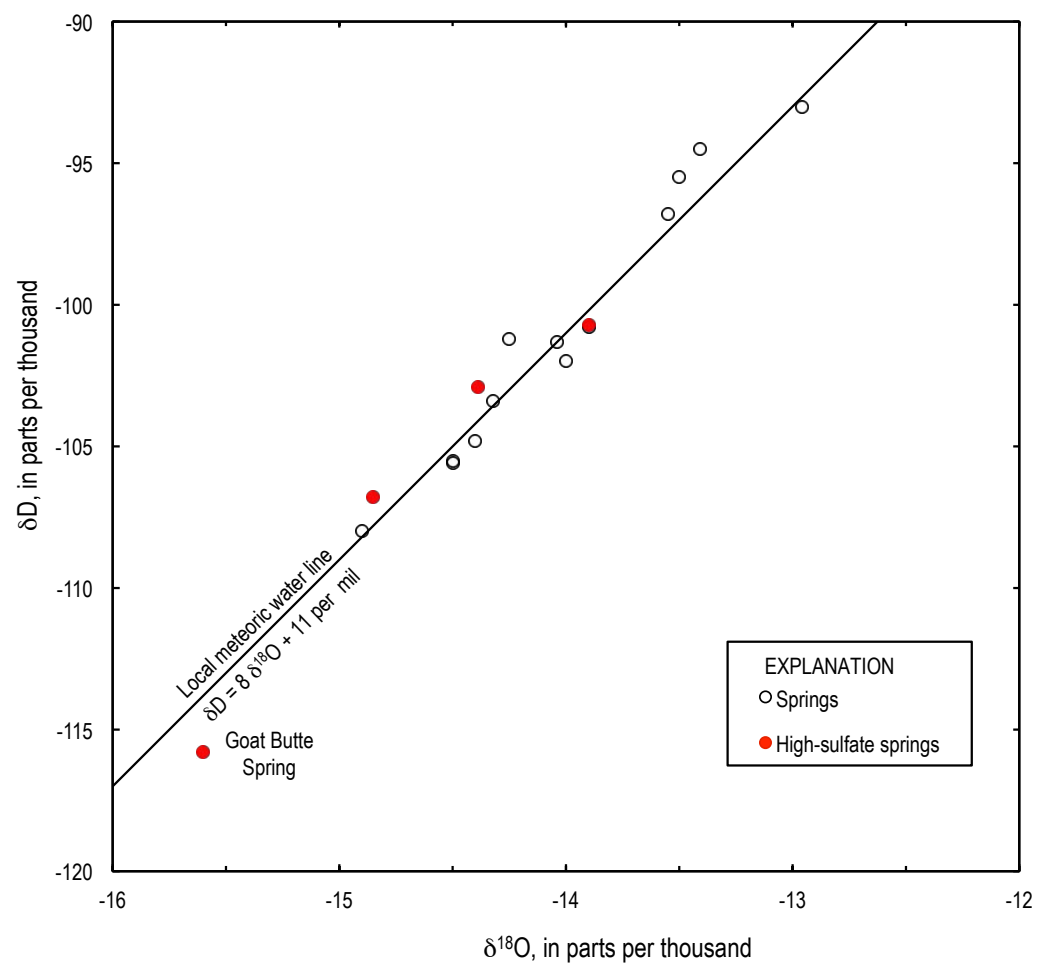

Figure 8. Graph of deuterium/hydrogen deviation $(\delta D)$ versus oxygen-18/oxygen-16 deviation $\left(\delta^{18} 0\right)$, both from Vienna Standard Mean Ocean Water, for water samples from table 2. Local meteoric water line determined by fixing slope at a value of 8 and calculating the intercept as the average of $\delta D-8 \delta^{18} 0$.

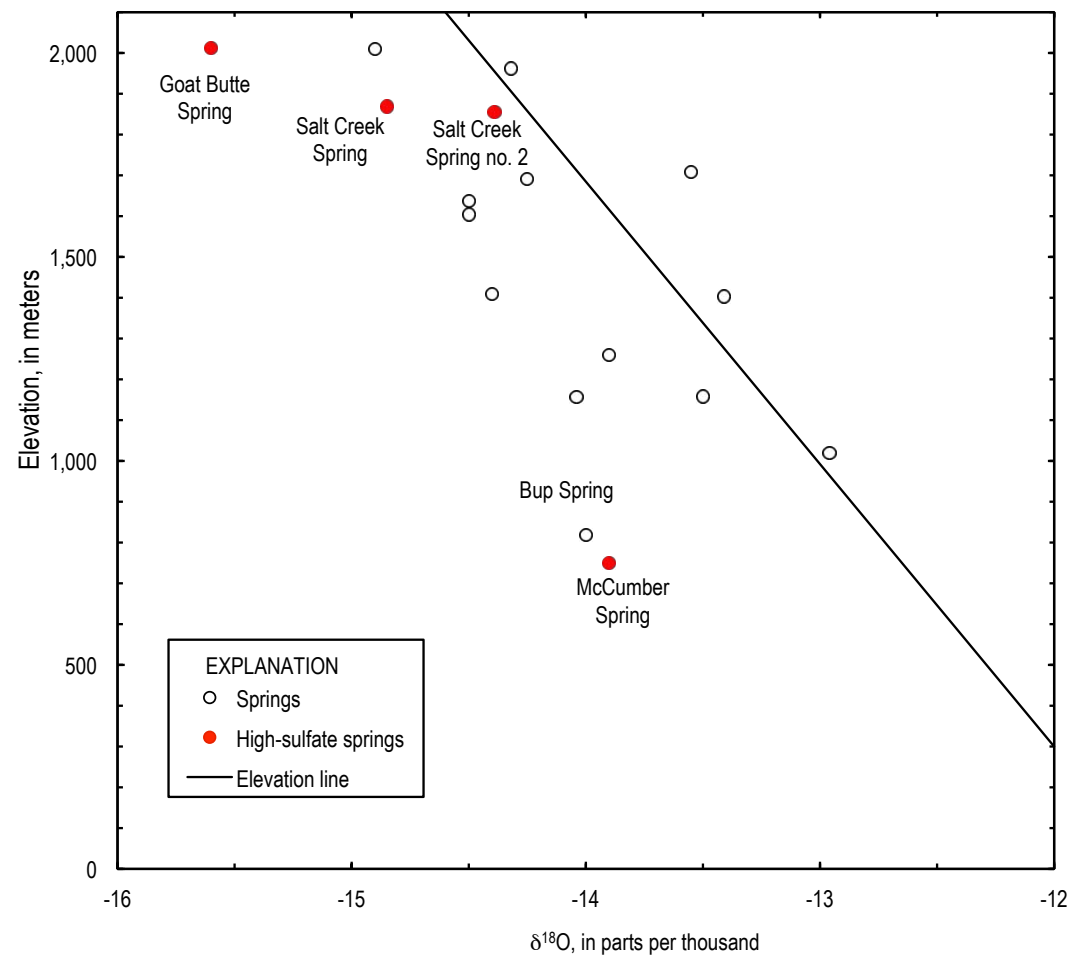

Figure 9. Graph of oxygen-18/oxygen-16 ratio deviation versus elevation for samples from table 2. 
Table 3. Chemical analysis of gas sample from the vent on Mount Adams, along with data for selected samples from Cascade volcanoes.

[Data for Mount Adams sample given both as measured in the sample and corrected for air contamination. Mount Adams gas vent located at $46^{\circ} 12.54^{\prime} 121^{\circ}$ 29.52' 3,609 m NAD27. Some analyses have data for additional constituents in original references. ${ }^{3} \mathrm{He} /{ }^{4} \mathrm{He}$ reported as ratio to value in air, and $\delta^{13} \mathrm{C}$ reported referenced to PeeDee belemnite (PDB). ", same as immediately above. Gas analysis by W.C. Evans and helium istopes by M.C. van Soest. Sample collected by R.H. Mariner and L.B. Christiansen.]

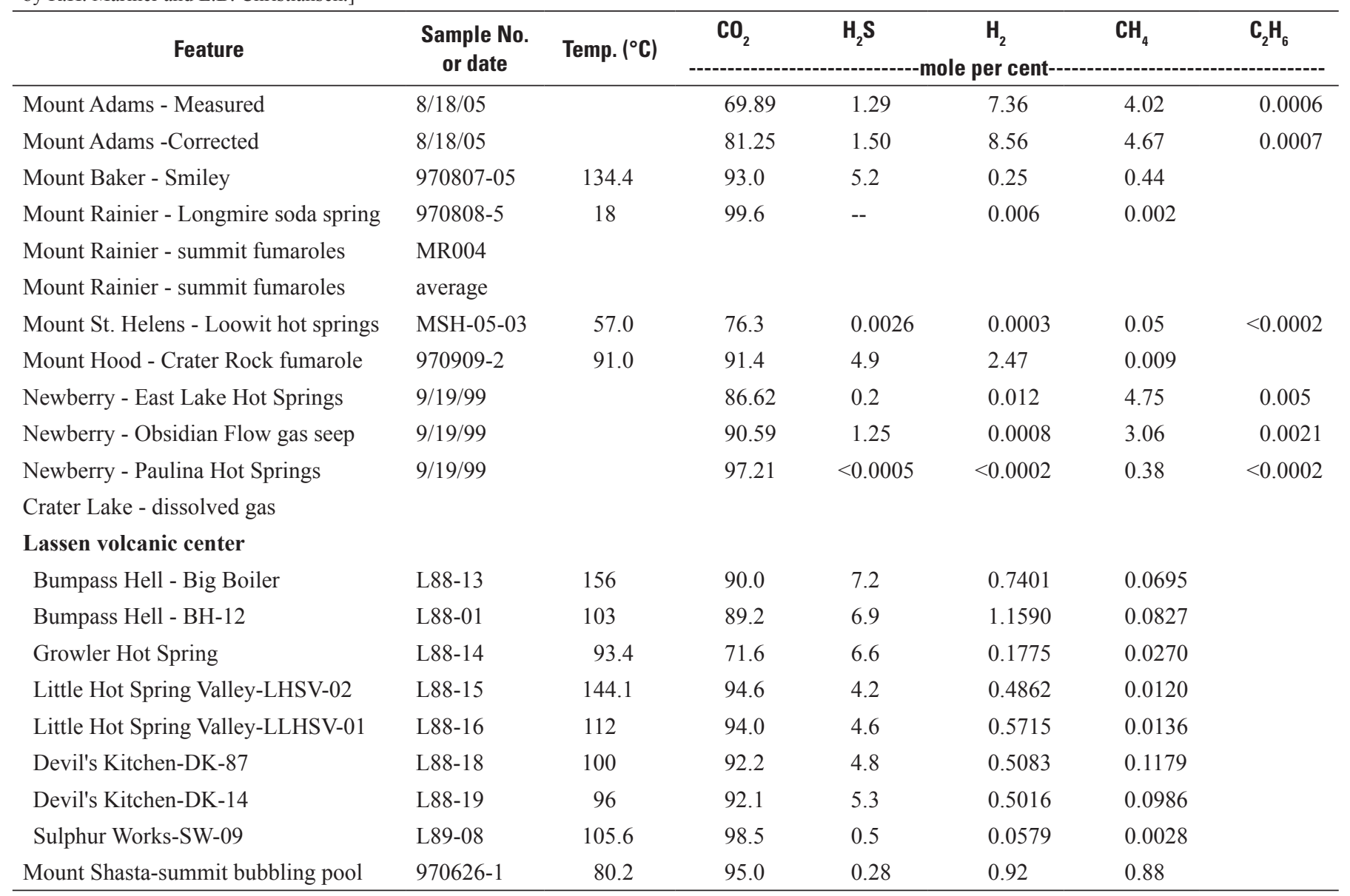

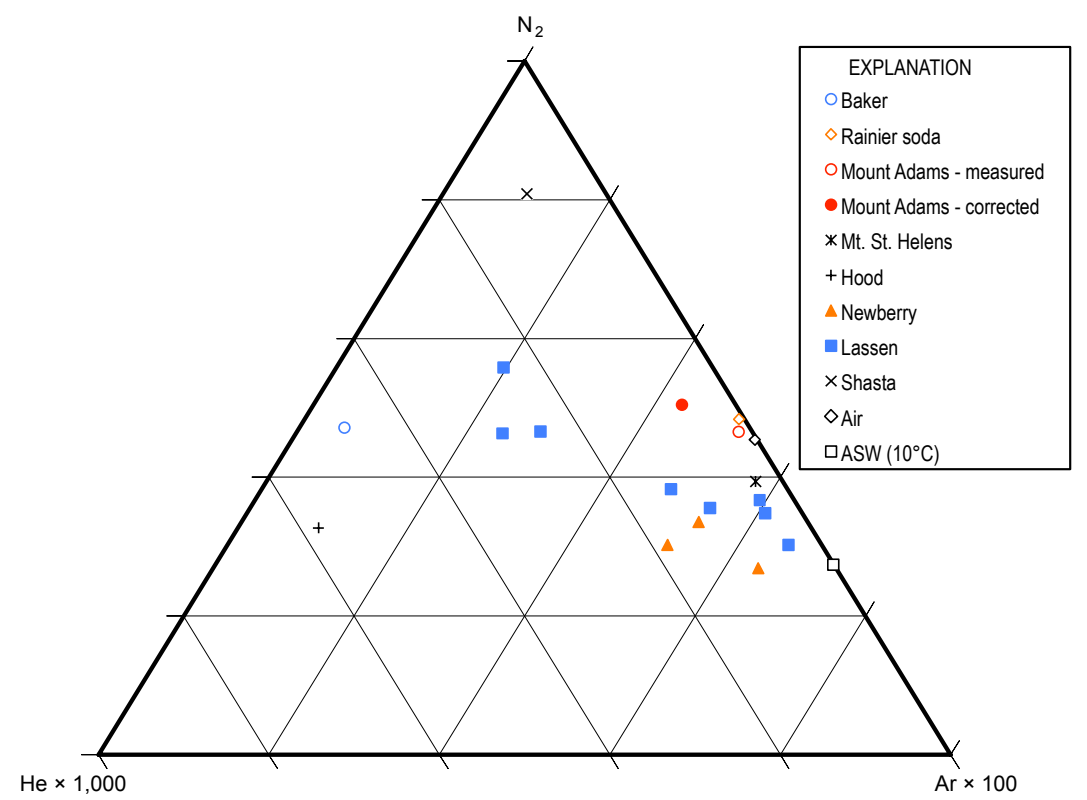

Figure 10. Triangular diagram showing relative proportions of nitrogen, argon, and helium for gas data from table 3. Relative proportions in air-saturated water (ASW) from solubility data in Wilhelm and others (1977). Diagram created using software from Graham and Midgley (2000). 
Table 3. Chemical analysis of gas sample from the vent on Mount Adams, along with data for selected samples from Cascade volcanoes.-Continued

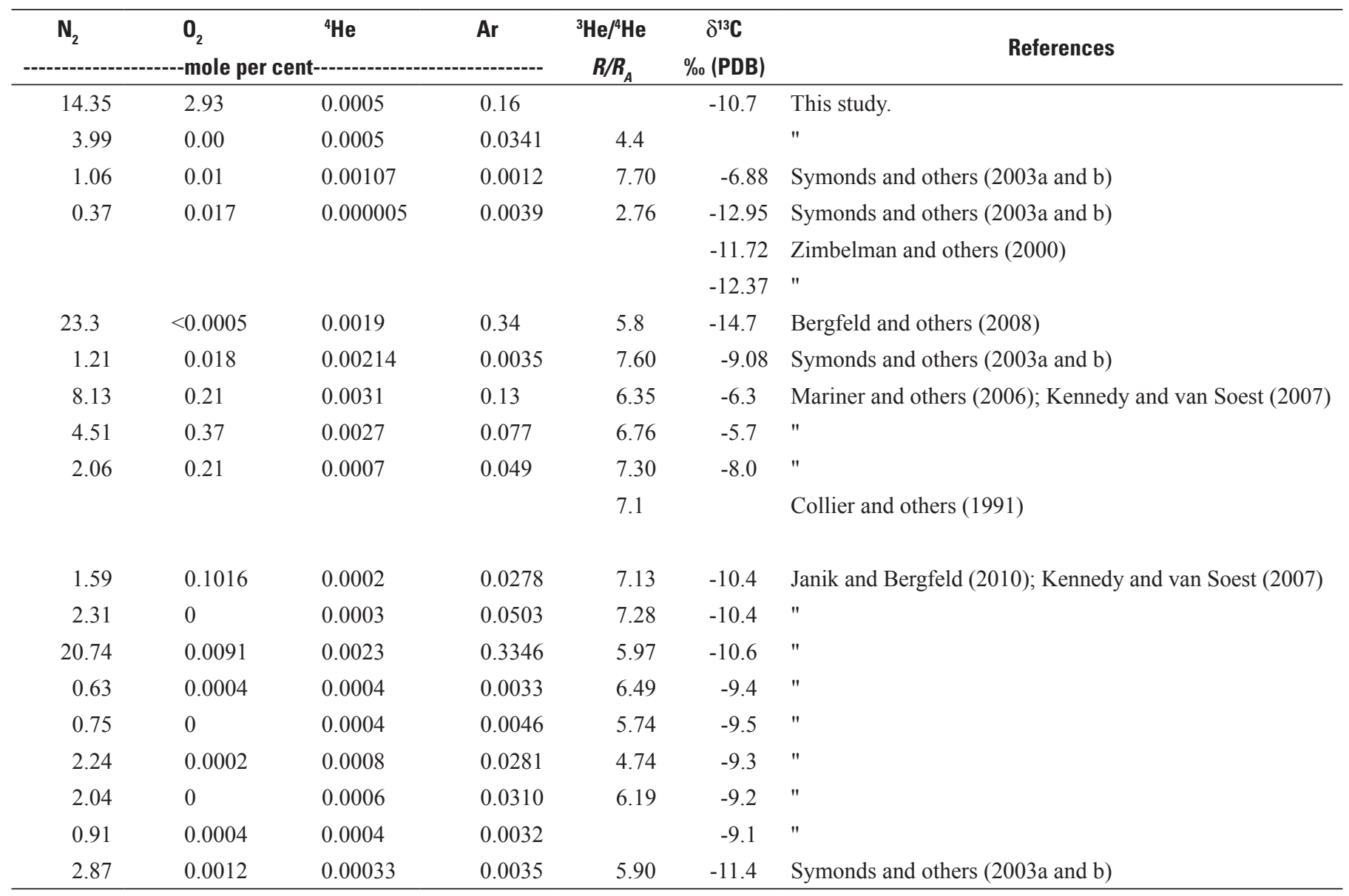

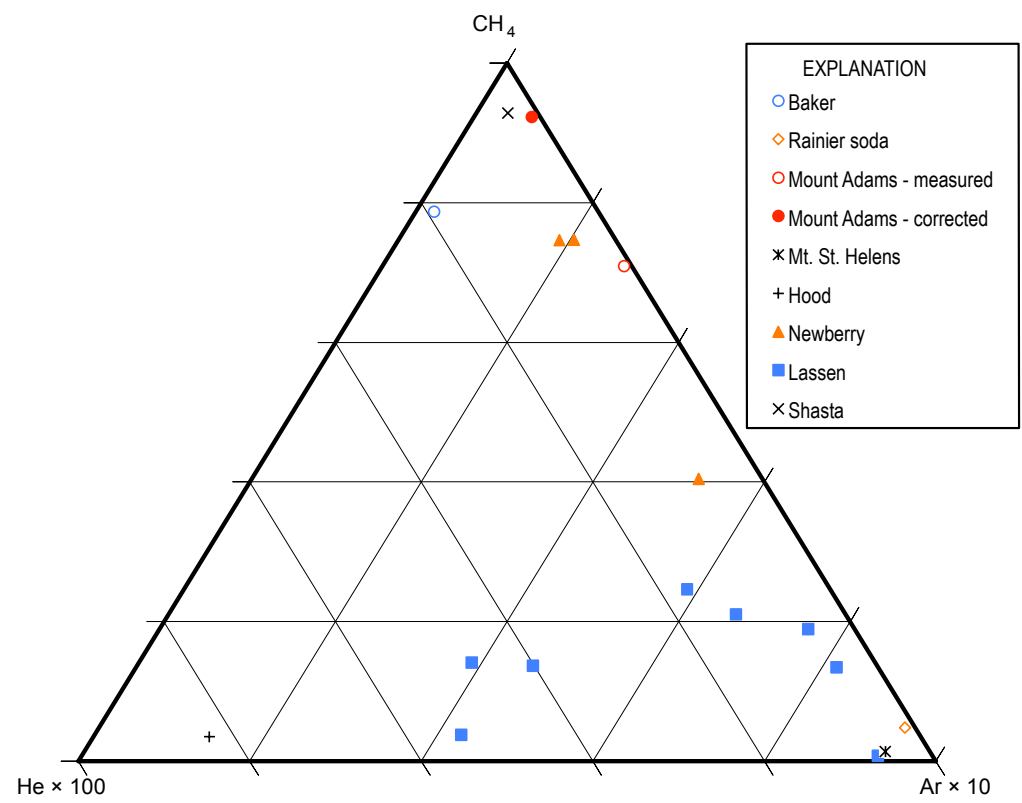

Figure 11. Triangular diagram showing relative proportions of methane, argon, and helium for gas data from table 3. Diagram created using software from Graham and Midgley (2000). 
However, as sulfate concentrations increase in the high sulfate springs, the specific conductance increases, and silica is less than what one expects if the specific conductance were all from bicarbonate. Salt Creek Spring no. 2 has the highest concentration of sulfate of any spring and is displaced the farthest from the other springs. The samples for Klickitat River and Big Muddy Creek behave similarly to the three high-sulfate spring samples in being only somewhat displaced from the line. The samples for Cascade Creek and Adams Creek have no bicarbonate, and their specific conductance is almost completely from dissolved sulfate from hydrothermal minerals. They also have low values of silica, because the dissolution of hydrothermal minerals is not very effective in adding silica compared to the action of dissolved carbon dioxide.

The chemistry of the high-sulfate samples is similar to that found in studies of waters draining mineralized areas (Verplanck and others, 2009). In such areas, sulfate is generally derived from the dissolution of gypsum $\left(\mathrm{CaSO}_{4} \cdot 2 \mathrm{H}_{2} \mathrm{O}\right)$ or from the decomposition of pyrite or other sulfide minerals in water to produce sulfur and sulfuric acid. Figure 6 plots calcium versus sulfate for our samples and also shows the line representing gypsum dissolution. Most springs have calcium concentrations that are independent of the sulfate concentrations, but Goat Butte and Salt Creek Spring waters are on the gypsum dissolution line. Klickitat River, Big Muddy Creek, and McCumber Spring appear to have derived calcium from both gypsum dissolution and the weathering of volcanic rock. Adams Creek, Cascade Creek, and Salt Creek Spring no. 2 appear to derive their sulfate primarily from the reaction of pyrite. Adams Creek and Cascade Creek have high iron (2.33 and $1.51 \mathrm{mg} / \mathrm{L}$, respectively), and this is consistent with their sulfate being from the reaction of pyrite. Unfortunately, we do not have data for iron in Salt Creek Spring no. 2. Cascade Creek water is quite cloudy, and the wetted area of rocks and sticks have a notable reddish-brown color (fig. 7). Copper and aluminum are also elevated in Adams Creek (table 2). Although most of the constituents are relatively dilute compared to water from areas draining ore deposits, iron and manganese in Adams and Cascade Creeks are above the secondary standards for drinking water (U.S. Environmental Protection Agency, 2011).

\section{Isotopic Composition of Springs}

Because the isotopic composition of precipitation varies with elevation (Dansgaard, 1964), data on light stable isotopes of spring water can be used to estimate the elevations of recharge areas. Figure 8 presents the light stable isotope data for the springs, reported as per mil deviations from Vienna Standard Mean Ocean Water (VSMOW) (Gonfiantini, 1978). The intercept of the meteoric water line shown in figure 8 has been adjusted to fit the data. These data do not cover a sufficient range to determine both a slope and an intercept different from the global meteoric water line, but adjusting the intercept from +10 per mil to +11 per mil is well determined.
The oxygen isotope data are plotted versus the elevation of the springs in figure 9. The isotopic composition of precipitation decreases (more negative values) with increasing elevation (Dansgaard, 1964). Assuming that some springs are recharged at elevations only somewhat above their discharge elevations, the samples having the highest $\delta^{18} \mathrm{O}$ values for a given elevation are used to define the line for local precipitation (fitted by eye) in figure 9 (Ingebritsen and others, 1992; Rose and others, 1996). Part of the fitting process involves making the lines for $\delta^{18} \mathrm{O}$ and $\delta \mathrm{D}$ (not shown) consistent with the local meteoric water line of figure 8 . The slope of the $\delta^{18} \mathrm{O}$ line is -1.4 per $\mathrm{mil} / \mathrm{km}$, less than the value of -2.3 per $\mathrm{mil} / \mathrm{km}$ found by Rose and others (1996) for the area north of Lassen Peak, but it is the same as the slope found for Mount Shasta of -1.4 per mil/ $\mathrm{km}$ (Nathenson and others, 2003) and similar to that for Mount Hood of -1.7 per mil/km (Nathenson, 2004).

Some of the springs are significantly lighter in isotopic composition compared to the isotope-elevation line, indicating that the water fell as precipitation at higher elevations before recharging the groundwater system. A number of springs appear to be fed by precipitation from a few hundred meters higher in elevation than their discharge elevation (fig. 9). Goat Butte, Bup, and McCumber Springs have inferred recharge elevations around $900 \mathrm{~m}$ above their discharge elevations. The higher sulfate Salt Creek Spring no.2 has heaver isotopes than the lower sulfate Salt Creek Spring. If they are related by mixing of a higher sulfate water with a dilute spring water, the diluting water is from a higher elevation than the high-sulfate water. The two springs are at similar elevations but separated by about $250 \mathrm{~m}$ and two intervening drainages. More likely than the mixing of two waters is that the springs have different circulation paths.

\section{Gas Vent}

A seeping gas vent in a small disturbed area near Adams Glacier was sampled in 2005 (table 3). A thermometer was not available during the sampling, but the gas was close to ambient temperature. The analysis of the gas showed significant oxygen, indicating that there was contamination with atmospheric air, and a recalculated analysis based on removing the oxygen and other atmospheric gases in proportion is given in table 3. The composition of the corrected analysis has high carbon dioxide and hydrogen sulfide, indicating that the gas is like that found in hydrothermal systems at Cascade volcanoes. The presence of hydrogen sulfide in the gas explains reports of hydrogen sulfide odor in the summit area (Hildreth and others, 1983). The gas geothermometer of D'Amore and Panichi (1980), based on amounts of hydrogen sulfide, hydrogen, methane, and carbon dioxide, yields a temperature of $282^{\circ} \mathrm{C}$, indicating a high-temperature hydrothermal system as the source of the gas. However, the lack of fumaroles on Mount Adams and the ambient temperature of the vent gas indicates that the gas is probably from a previously active system rather than one that is currently active. 
The gas analysis from Mount Adams has both similarities to and differences from analyses from other Cascade volcanoes (table 3 ). The sample as originally analyzed plots near the composition for air on the nitrogen, argon, and helium triangular diagram (fig. 10). The corrected sample analysis is still close to the composition for air. In contrast, the various samples for the diverse parts of the Lassen hydrothermal systems define an array of values trending towards the value for air-saturated water (Janik and McLaren, 2010). The analyses for Mounts Shasta, Baker, and Hood define a range of values, but all show a relatively low value for the fraction of argon, and the sample for Sulphur Works at Lassen is close to this range. The array of values for Lassen show an increasing proportion of gas from air-saturated water. The samples from Newberry also plot close to the value for air saturated water. Conversely, the sample from Longmire soda spring, low on the flank of Mount Rainier, is also close to the composition of air rather than that of air-saturated water.

The gas from the Mount Adams vent has a high proportion of methane (fig. 11), as does that from Mount Baker, Mount Shasta, and two of the samples from Newberry. Other Cascade volcanoes have lower amounts of methane (fig. 11). Helium isotopes for the gas from Mount Adams are elevatedthe ${ }^{3} \mathrm{He} /{ }^{4} \mathrm{He}$ ratio, $\mathrm{R}$, is 4.4 times its value in the atmosphere, $\mathrm{R}_{\mathrm{A}}$, indicating a mantle source for some of the helium. But that ratio is lower than most of the values in table 3 from other Cascade volcanoes, indicating that there has been some mixture with crustal helium. (The corrected gas analysis may still contain some constituents from air. Helium isotopes are corrected for air contamination using a different methodology that should remove any helium that came from air.) Typical values for $\delta^{13} \mathrm{C}$ for carbon dioxide from the mantle range from -5 to -8 per mil (Symonds and others, 2003b). Mount Baker and Newberry Volcano have for $\delta^{13} \mathrm{C}$ values for carbon dioxide that are within this range, whereas values for Mount Adams and other volcanoes are more negative (table 3 ). The more negative values of $\delta^{13} \mathrm{C}$ in carbon dioxide in Cascade volcanic gases could mean that carbon from sediments subducted into the mantle is providing some of the carbon dioxide in basaltic parent magma (Evans and others, 2010), or they could mean that some of the carbon dioxide has a source from recent sediments mixing with mantle-derived carbon dioxide (Symonds and others, 2003b).

\section{Discussion}

The water chemistry of most springs on Mount Adams is a result of the weathering of volcanic rock. Some springs have added sulfate from the dissolution of hydrothermal minerals, including gypsum, or by reaction with sulfides. Some streams also have added sulfate-Adams and Cascades Creeks have no bicarbonate, added sulfate, and high iron. The gas sample from Mount Adams appears to reflect processes from a hightemperature $\left(200^{\circ}-300^{\circ} \mathrm{C}\right)$ hydrothermal system. There are no obvious fumaroles on Mount Adams, nor did the gas sample have an anomalous temperature. We conclude that the lack of any signature of thermal water at Mount Adams, together with the lack of high-temperature degassing, indicates that there is no active hydrothermal system. Thus the gas sample reflects a previous hydrothermal system that is no longer active. This is surprising, given the evidence for intense hydrothermal activity in the past. Of the major Cascade volcanoes, Mount Adams is the only one with no evidence for present hydrothermal discharge (Ingebritsen and Mariner, 2010).

\section{Acknowledgments}

Permission to do field work on the lands of the Yakama Nation in 2004 was facilitated by Scott Ladd and Warren Niemi. Judy Windt provided field assistance. Deb Bergfeld and Jake Lowenstern are thanked for helpful reviews.

\section{References Cited}

Bergfeld, Deborah, Evans, W.C., McGee, K.A., and Spicer, K.R., 2008, Pre- and post-eruptive investigations of gas and water samples from Mount St. Helens, Washington, 2002 to 2005, chap. 25 of Sherrod, D.R., Scott, W.E., and Stauffer, P.H., eds., A volcano rekindled; the renewed eruption of Mount St. Helens, 2004-2006: U.S. Geological Survey Professional Paper 1750, p. 523-542.

Cline, D.R., 1976, Reconnaissance of the water resources of the Upper Klickitat River Basin, Yakima Indian Reservation, Washington: U.S. Geological Survey Open-File Report $75-518,54 \mathrm{p}$.

Collier, R.W., Dymond, Jack, and McManus, J.R., 1991, Studies of hydrothermal processes in Crater Lake, OR: Oregon State University College of Oceanography Report 90-7, 201 p., appendices. (Also available at http://ir.library. oregonstate.edu/xmlui/handle/1957/14875?show=full.)

D'Amore, Franco, and Panichi, Costanzo, 1980, Evaluation of deep temperatures of hydrothermal systems by a new gas geothermometer: Geochimica et Cosmochimica Acta, v. 44, p. 549-556.

Dansgaard, W., 1964, Stable isotopes in precipitation: Tellus, v. 16, p. $436-468$.

Evans, W.C., Mariner, R.H., Bergfeld, D., Revesz, K.M., and McGeehin, J.P., 2010, Carbon isotope composition of $\mathrm{CO}_{2}$ at Cascade Arc volcanoes, in Birkle, Peter, and TorresAlvarado, I.S., eds., Water-rock interaction, Proceedings 13th international conference on water-rock interaction, Guanajuato, Mexico, Aug. 16-20, 2010: London, CRC Press, p. 195-198 
Finn, C.A., Deszcz-Pan, Maryla, Anderson, E.D., and John, D.A., 2007, Three-dimensional geophysical mapping of rock alteration and water content at Mount Adams, Washington-Implications for lahar hazards: Journal of Geophysical Research, v. 112, B10204, doi:10.1029/2006JB004783, $21 \mathrm{p}$.

Fretwell, M.O., 1979, Quality of surface and ground waters, Yakima Indian Reservation, Washington, 1973-74: U.S. Geological Survey Open-File Report 77-128, 177 p., 2 plates, scale 1:250,000.

Gonfiantini, R., 1978, Standards for stable isotope measurements in natural compounds: Nature, v. 271, p. 534-536.

Graham, D.J., and Midgley, N.G., 2000, Graphical representation of particle shape using triangular diagrams; an Excel spreadsheet method: Earth Surface Processes and Landforms, v. 25, p. 1473-1477, accessed October 3, 2011, at http://www.lboro.ac.uk/research/phys-geog/tri-plot/index. html.

Hildreth, Wes, and Fierstein, Judy, 1995, Geologic map of the Mount Adams volcanic field, Cascade Range of southern Washington: U.S. Geological Survey Miscellaneous Investigations Series I-2460, 39 p., 1 plate, scale 1:50,000.

Hildreth, W., and Fierstein, J., 1997, Recent eruptions of Mount Adams, Washington Cascades, USA: Bulletin of Volcanology, v. 58, p. 472-490.

Hildreth, W., and Lanphere, M.A., 1994, Potassium-argon geochronology of a basalt-andesite-dacite arc system-The Mount Adams volcanic field, Cascade Range of southern Washington: Geological Society of America Bulletin, v. 106, p. 1413-1429.

Hildreth, Wes, Fierstein, Judy, and Miller, M.S., 1983, Mineral and geothermal resource potential of the Mount Adams wilderness and contiguous roadless areas, Skamania and Yakima Counties, Washington: U.S. Geological Survey Open-File Report 83-474, 35 p., accessed April 8, 2013, at http://pubs.er.usgs.gov/publication/ofr83474.

Ingebritsen, S.E., and Mariner, R.H., 2010, Hydrothermal heat discharge in the Cascade Range, northwestern United States: Journal of Volcanology and Geothermal Research, v. 196, p. 208-218.

Ingebritsen, S.E., Sherrod, D.R., and Mariner, R.H., 1992, Rates and patterns of groundwater flow in the Cascade Range volcanic arc, and the effect on subsurface flow: Journal of Geophysical Research, v. 97, p. 4599-4627.

Janik, C.J., and Bergfeld, Deborah, 2010, Analyses of gas, steam and water samples collected in and around Lassen Volcanic National Park, California, 1975-2002: U.S. Geological Survey Open-File Report 2010-1036, 13 p., accessed February 1, 2012, at http://pubs.usgs.gov/of/2010/1036/.
Janik, C.J., and McLaren, M.K., 2010, Seismicity and fluid geochemistry at Lassen Volcanic National Park, California; Evidence for two circulation cells in the hydrothermal system: Journal of Volcanology and Geothermal Research, v. 189 , p. $257-277$.

Kennedy, B.M., and van Soest, M.C., 2007, Flow of mantle fluids through the ductile lower crust-Helium isotope trends: Science, v. 318, p. 1433-1436.

Mariner, R.H., Venezky, D.Y., and Hurwitz, S., 2006, Chemical and isotopic database of water and gas from hydrothermal systems with an emphasis for the western United States: U.S. Geological Survey Data Series 169, data base, accessed February 1, 2012, at http://pubs.usgs.gov/ ds/2005/169/.

Nathenson, Manuel, 2004, Springs on and in the vicinity of Mount Hood volcano, Oregon: U.S. Geological Survey Open-File Report 2004-1298, 43 p. (Also available at http:// pubs.usgs.gov/of/2004/1298/.)

Nathenson, Manuel, and Thompson, J.M., 1990, Chemistry of Crater Lake, Oregon, and nearby springs in relation to weathering, in Drake, E.T., Larson, G.L., Dymond, J., and Collier, R., eds., Crater Lake, an ecosystem study: San Francisco, American Association for the Advancement of Science, Pacific Division, p. 115-126.

Nathenson, M., Thompson, J.M., and White, L.D., 2003, Slightly thermal springs and non-thermal springs at Mount Shasta, California; Chemistry and recharge elevations: Journal of Volcanology and Geothermal Research, v. 121, p. 137-153.

National Oceanic and Atmospheric Administration, 1997a, Climatological data annual summary Oregon 1997: Asheville, N.C., National Climatic Data Center, v. 103, no. 13, $32 \mathrm{p}$.

National Oceanic and Atmospheric Administration, 1997b, Climatological data annual summary Washington 1997: Asheville, N.C., National Climatic Data Center, v. 101, no. $13,25 \mathrm{p}$.

Reed, M.J., 1983, Introduction, in Reed, M.J., ed., Assessment of low-temperature geothermal resources of the United States-1982: U.S. Geological Survey Circular 892, p. 1-8.

Rose, T.P., Davisson, M.L., and Criss, R E., 1996, Isotope hydrology of voluminous cold springs in fractured rock from an active volcanic region, northeastern California: Journal of Hydrology, v. 179, p. 207-236.

Symonds, R.B., Janik, C.J., Evans, W.C., Ritchie, B.E., Counce, Dale, Poreda, R.J., and Iven, Mark, 2003a, Scrubbing masks magmatic degassing during repose at CascadeRange and Aleutian-Arc volcanoes: U.S. Geological Survey Open-File Report 03-435, 22 p. (Also available at http:// geopubs.wr.usgs.gov/open-file/of03-435/.) 
Symonds, R.B., Poreda, R.J., Evans, W.C., Janik, C.J., and Ritchie, B.E., 2003b, Mantle and crustal sources of carbon, nitrogen, and noble gases in Cascade-Range and AleutianArc volcanic gases: U.S. Geological Survey Open-File Report 03-436, 26 p. (Also available at http://geopubs. wr.usgs.gov/open-file/of03-436/.)

U.S. Environmental Protection Agency, 2011, Secondary drinking water regulations; Guidance for nuisance chemicals: accessed July 25, 2011, at http://water.epa.gov/drink/ contaminants/secondarystandards.cfm.

Vallance, J.W., 1999, Postglacial lahars and potential hazards in the White Salmon River system on the southwest flank of Mount Adams, Washington: U.S. Geological Survey Bulletin 2161, 49 p., 2 plates, scale 1:100,000.

Verplanck, P.L., Nordstrom, D.K., Bove, D.J., Plumlee, G.S., and Runkel, R.L., 2009, Naturally acidic surface and ground waters draining porphyry-related mineralized areas of the Southern Rocky Mountains, Colorado and New Mexico: Applied Geochemistry, v. 24, p. 255-267.

Western Regional Climate Center, 2011a, Oregon Climate Summaries: Desert Research Institute, accessed March 25, 2011, at http://www.wrcc.dri.edu/summary/climsmor.html.

Western Regional Climate Center, 2011b, Washington Climate Summaries: Desert Research Institute, accessed March 25, 2011, at http://www.wrcc.dri.edu/summary/climsmwa.html.

Wilhelm, Emmerich, Battino, Rubin, and Wilcock, R.J., 1977, Low-pressure solubility of gases in liquid water: Chemical Reviews, v. 77, p. 219-262.

Zimbelman, D.R., Rye, R.O., and Landis, G.P., 2000, Fumaroles in ice caves on the summit of Mount Rainier-preliminary stable isotope, gas, and geochemical studies: Journal of Volcanology and Geothermal Research, v. 97, p. 457-473. 
흠

z

3

三

흘

음

。

옹

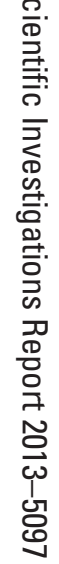

